\title{
Studies on the Phyto-Toxicity of Heavy Metal Mercury in Wheat (Triticum AestivumL) I-Germination Percentage (GP) and Speed of Germination Index (SGI)
}

\author{
Kapila Saraswat ${ }^{1}$, Nidhi Parashar ${ }^{2}$, Snehlata $^{1}$, Abha Pathak ${ }^{1}$ and Ravi Sharma ${ }^{1 *}$ \\ ${ }^{1}$ Department of Botany, KR College, Mathura, Dr BR Ambedkar University, formerly Agra University, India
}

${ }^{2}$ Department of Chemistry KR College, Mathura, Dr BR Ambedkar University, formerly Agra University, India

*Corresponding author: Ravi Sharma,Formerly Head Department of Botany, KR College, Mathura and Ex-Founder Principal ESS College of Education, Agra327/Sec-9 Avas Vikas Colony Sikandra Agra 282007 India

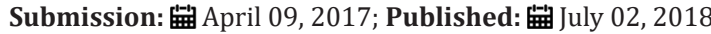

\begin{abstract}
In the present investigation studies were made on the Phyto-toxicity of Heavy Metal Mercury (Hg) in four Wheat (Triticum aestivumL) varieties taking into consideration Germination Percentage (GP) and Speed of Germination Index (SGI) to bring out varietal tolerance behavior. It was observed that the germination decreased in the four wheat varieties viz., V1 Lok-1, V2 UP-2338, V3 PBW-154 and V4 PBW-502 with increasing concentration of Hg. A comparison with controls in the four varieties recorded in the controls: at 24 hours GP was lowest in the variety V1 (23.66\%) and highest in V4 (26.66\%); at 48 hours lowest in V1 (51.0\%) and highest in V4 (54.66\%); at 72 hours lowest in V1 (61.33\%) and highest in V3 (66.66\%); at 96 hours lowest in V2 (82.0\%) and highest in V3 and V4 (84.33\%) and at 120 hours lowest in V2 (86.0\%) and highest in V3 (94.0\%) respectively. On the other hand, in 100, 200 and 300ppm of mercury concentration treatments there was no germination found at all at 24 hours in all the four varieties while in 100 ppm at 48 hours lowest GP was in V2 (16.0\%) and highest in V3 and V4 (21.33\%); at 72 hours lowest in V4 (31.33\%) and highest in V2 (36.0\%); at 96 hours lowest in V1 (44.0\%) and highest in V2 (49.0\%) and at 120 hours lowest in V4 (51.0\%) and highest in V2 (53.66\%) respectively. In 200ppm Hg concentration at 48 hours lowest GP was found in V4 (9.66\%) and highest in V3 (13.66\%) respectively; at 72 hours lowest in V4 (25.66\%) and highest in V1 (37.0\%); 96 hours lowest in V4 (37.33\%) and highest in V1 (43.0\%) and at 120 hours lowest in V3 (42.0\%) and highest in V4 (48.33\%) respectively. Lastly, in 300ppm at 48 hours lowest GP was in V4 (3.0\%) and highest in V1 (5.0\%); at 72 hours lowest in V2 (8.0\%) and highest in V1 (12.0\%); 96 hours lowest in V4 (17.0\%) and highest in V1 (21.66\%) and at 120 hours lowest in V2 (26.0\%) and highest in V1 (32.33\%) respectively. A comparison with controls in the four varieties recorded maximum average GP (65.13\%) in control sets of var. V3 whereas minimum (11.2\%) was recorded in the 300ppm $\mathrm{Hg}$ treatment sets in the var. V2. On the other hand, data on the basis of percent over control showed maximum GP (48.98\%) in the var. V2 in 100ppm Hg treatment and the minimum (18.0\%) was also recorded in the var. V2 in 300ppm Hg treatment. Showing a clear cut maximum decrease of (-81.99\%) in the var. V2 in 300ppm Hg treatment and also minimum decrease of (-51.02\%) in the var. V2 in 100ppm Hg treatment. With this SGI showed the trend as highest (811.66) in controls of V3 while lowest of (102.0) in V2 in 300ppm Hg treatment while data on percent over control basis showed highest (43.08) in 100ppm Hg treatment in the var. V1 while lowest (13.03) in 300ppm Hg treatment in the var. V2. Showing maximum decrease of (-86.96) in the var. V2 in 300ppm Hg treatment and minimum decrease of (-56.91) in the var. V1 in 100ppm Hg treatment.
\end{abstract}

Keywords:Wheat varieties; Germination percentage (GP); Speed of germination index (SGI); Mercury toleranc

\section{Introduction}

Unfortunately, land and water, the two precious natural resources on which relies the sustainability of agriculture and the civilization of mankind have been subjected to maximum exploitation and severely degraded/polluted due to anthropogenic activities. The pollution includes point sources such as emission [1-3], effluents and solid discharge from industries [4-7], vehicle exhaustion [1-2]. and metals from smelting and mining, and non-point sources such as soluble salts (natural and artificial) [8-14], use of insecticides/pesticides [15], disposal of industrial and municipal wastes in agriculture $[7,16]$, and excessive use of fertilizers [17-19]. Contamination of agricultural land caused by heavy metal in and around industrial areas is a serious problem. According to several scientists such contamination is largely due to injudicious anthropogenic activities such as indiscriminate use of pesticides containing heavy metals in agriculture, discharge of untreated industrial wastes and effluents, faulty waste disposal, high rate of burning of fossil fuels, mining etc. [20-24].

Each source of contamination has its own damaging effects to plants, animals and ultimately to human health, but those that add heavy metals to soils and waters are of serious concern due 
to their persistence in the environment and carcinogenicity to human beings. They cannot be destroyed biologically but are only transformed from one oxidation state or organic complex to another $[25,26]$. Therefore, heavy metal pollution poses a great potential threat to the environment and human health [4-7,15,16,27-29].

Though, Lead is a ubiquitous poison known to man from biblical times Mercury became notorious in the recent past as a result of Minamata disease in Japan and has been found guilty as a harmful environmental pollutant. What worries us now and causes considerable concern is that this and other toxic metals are being found in an increasing amount in the human environment, in the air we breathe, in the water we drink and in the food we consume. Heavy metal toxicity, such as due to mercury in our environment, is seriously dangerous because these metals persist in the environment for years together [28]. The poor socio-economic conditions and lack of awareness in the agricultural community towards the hazards associated with waste water irrigation, it is practically impossible to put a stop to it. The magnitude of the danger of environmental pollution by heavy metal, especially mercury, was probably for the first time realized with the Minamata Disaster in Japan [6,28,30,31] when thousands of people suffered with mercury poisoning after consuming the fish caught in Minamata Bay contaminated by mercury released between 1953 and 1960 from the Chisso Corporation's Chemical Factory, Vinyl Chloride Plant [30]. Though, Minamata has made mercury famous but mercury has been around for a very long time. Metallic mercury in its liquid form is of little or no significance as a threat to health however, inhalation of metallic mercury vapours can cause acute or chronic health effects. Several of mercury salts are extremely toxic, the best known of which is mercuric chloride $\left(\mathrm{HgCl}_{2}\right)$. Organic compounds of mercury are the ones which are causing the greatest concern. Mercury is bio-transformed to Zinc mercuric compounds. This has been considered to be responsible for the Minamata episode [30].

The mercury cycle is a bio-geochemical cycle involving mercury. Mercury is notable for being the only heavy metal which is liquid at room temperature. It is volatile metal and evaporates, though it takes quite a while to do so. Most natural mercury occurs as cinnabar (HgS) where mercury $\left(\mathrm{Hg}^{2+}\right)$ is bound very tightly to sulphur but weathering slowly releases the mercury to the environment [32]. There are also trace amounts of mercury in coal. Mining mercury or burning coal results in releasing mercury. Volcanoes and forest fires are also sources of mercury. Chlorine factories, among other sources, release mercury into the atmosphere. This mercury is deposited back onto land and water. Inorganic mercury can be converted by bacteria into the organo-metallic cation known as methyl mercury $\left(\mathrm{CH}_{3} \mathrm{Hg}^{+}\right)$which bio-accumulates in fish. Over long periods of time, some mercury recombines with sulphur and is buried in sediments.

Then, the cycle repeats itself. Briefly, the bio-geochemical cycle of $\mathrm{Hg}$ starts with the evaporation of $\mathrm{Hg}$ from natural and anthropogenic sources, which is then oxidized to inorganic Hg. This element is spread by the rain. Once in the soil, Hg can be transformed into organic compounds by bacteria. Fish and shellfish have a natural tendency to concentrate mercury in their bodies, often in the form of methyl mercury. Species of fish that are high on the food chain, such as shark, swordfish, etc., contain higher concentrations of mercury than others. As mercury and methyl mercury are fat soluble, they primarily accumulate in the viscera, although they are also found throughout the muscle tissue.

When this fish is consumed by a predator, the mercury level is accumulated. Since fish are less efficient at depurating than accumulating methyl mercury, fish-tissue concentrations increase over time. Thus species that are high on the food chain amass body burdens of mercury that can be ten times higher than the species they consume called bio-magnification. Mercury poisoning happened this way in Minamata [33] therefore, called Minamata disease. Further, microorganisms in the water convert mercury to this highly toxic form methyl mercury and thus bacteria makes the mercury "bio-available" to be transported to fish and then to man.

Land and water pollution by heavy metals is a worldwide issue. All countries have been affected, though the area and severity of pollution vary enormously. In Western Europe, 1,400,000 sites were affected by heavy metals [34], of which, over 300,000 were contaminated, and the estimated total number in Europe could be much larger, as pollution problems increasingly occurred in Central and Eastern European countries [35]. In USA, there are 600,000 brown fields which are contaminated with heavy metals and need reclamation [36]. According to statistics, coal mine has contaminated more than 19,000 km of US streams and rivers from heavy metals, acid mine drainage and polluted sediments.

More than 100,000 ha of cropland, 55,000 ha of pasture and 50,000 ha of forest have been lost [37]. The problem of land pollution is also a great challenge in China, where one-sixth of total arable land has been polluted by heavy metals, and more than $40 \%$ has been degraded to varying degree due to erosion and desertification [38]. Soil and water pollution is also severe in India, Pakistan and Bangladesh, where small industrial units are pouring their untreated effluents in the surface drains, which spread over near agricultural fields. In these countries raw sewage is often used for producing vegetables near big cities [14-16].

The development of the intensive agriculture between 1960 and 1990 totally over passed the aspect connected with the negative impact of the toxic chemical compounds on the air, water and soil. As one of the consequences of heavy metal pollution in soil, water and air, plants are contaminated by heavy metals. Using chemical products as nutrients, fertilizers and pesticides, we believe that we attack our safety and we must know the effects of heavy metals from these compounds. Many researchers examined the inhibitory effect of heavy metal compounds on growth and the performance of photosynthetic apparatus of plants. There are two aspects on the interaction of plants with heavy metals:

(i) Heavy metals show negative effects on plants.

(ii) Plants have their own resistance mechanisms against toxic effects and for detoxifying heavy metal pollution. 
The effects of heavy metals on plants resulted in growth inhibition, structural damage, decline of physiological and biochemical activities, as well as of the function of plants. The effect of different metallic ions in the hydrolysis of ATP is studied in. Carotenes especially have attracted much attention in recent years for their biological function. Knowing the nutrients required to grow plants is only one aspect of successful crop production. Optimum yield also requires knowing the rate to apply, the method and time of application, the source of nutrients to use, and how the elements are influenced by soil and climatic conditions.

Mercury is an environmental pollutant which is mainly supplied via anthropogenic sources to the soil. It is harmful because of its toxicity, mobility, bio-accumulation, methylation process and transport in the atmosphere [39]. Plants living in Hg-enriched ecosystems can be adapted to field conditions by detoxification mechanisms, although $\mathrm{Hg}$ is a metal without known biological function in higher plants. Hg compounds are highly toxic to plants and concentrations in plant tissues increase with age. Soil $\mathrm{Hg}$ availability for plants is usually low because it is absorbed in the soil or precipitated in the soil solution and it is mainly accumulated in roots [40]. Nevertheless it reaches the shoots by translocation or foliar absorption. The most common chemical species in soils are $\mathrm{Hg}^{0}$ and $\mathrm{Hg}^{++}$. Long range atmospheric transport of mercury, in large part from coal combustion, can contribute up to $50 \%$ of total loading to humus rich soils. Humic matter forms strong complexes with $\mathrm{Hg}^{++}$. In fact, transport of $\mathrm{Hg}$ in soil water is largely due to association with soluble humic matter. Chronic exposure due to consumption of methyl mercury in fish and other sea food with subsequent neurotoxicity is a human health concern [41].

Methyl mercury forms in anaerobic sediments of aquatic ecosystems and biomagnifies through tropic transfer to fish. The adverse effects of toxic chemicals on soil fauna and microbes are of the major foci in soil eco-toxicological assessments. There are several kinds of standardized plant toxicity tests, i.e., seed germination, root elongation and early seedling growth tests. Photosynthesis inhibition test and enzyme content fluctuation are also frequently used as endpoints for phyto-toxicity. The present study selected wheat for testing as it is the main staple cereal in the world.

Furthermore, germination (GP), speed of germination (SGI), root elongation is selected as a quantitative test endpoint in this study as the root accumulate more toxicants and is more sensitive than shoot. Recent reports on the toxic effects of heavy metals on wheat indicate that heavy metals inhibit GP and SGI with root and shoot growth $[5,7,14,16,28,42,43]$ and also induce oxidative stress and lipid per-oxidation $[5,14,28,44]$. Various defense mechanisms adopted by wheat to avoid heavy metal toxicity have been reported by several researchers.

These include: alteration of antioxidant enzyme level $[5,14,28,43-45]$ increase in the content of phyto-chelation $[46,47]$ and increase generation of polyamine and ethylene [48]. High concentrations of heavy metals in soil can negatively affect crop growth, as these metals interfere with metabolic functions in plants, including physiological and biochemical processes [5,14,28,], inhibition of photosynthesis, and respiration and degeneration of main cell organelles, even leading to death of plants $[25,49,50]$. In order to cope up with heavy metal contaminated soils, various phyto-remediation approaches (phyto-stabilization, phyto-immobilization and phyto-extraction) can be applied. However, the choice will depend on many factors, such as plant tolerance to pollutants, soil physic-chemical properties, agronomic characteristics of the plant species, climatic conditions (rainfall, temperature), and additional technologies available for the recovery of metals from the harvested plant biomass. It appears that both chemical and biological approaches are passing through their infancy and need more efforts for their effective use in the future $[5,7,14,16,27,28]$.

\section{Significance of the Study}

Environmental Pollution has emerged as a major epidemic endangering Life on earth. Due to unwise, unscientific and excessive use of natural resources eco-balance is disturbed. Industrialization and urbanization have also deteriorated the position. As such pollution can be considered the result of the growth of modern civilization. Hence effective Pollution control is the need of the hour. Episodes like the Minamata and Itai-Itai epidemics in Japan serve as a warning against the indiscriminate and careless use of toxic heavy metals. For effective control of heavy metal pollution, it is necessary to continuously monitor the environment for their presence, to initiate a system for biological monitoring for heavy metal exposure and to take appropriate steps to minimize and control heavy metal pollution.

\section{Objectives}

Thus, the objective of this work was to evaluate the toxicity of heavy metal mercury on wheat crop to provide information on the significance of seed GP and SGI, root/shoot ratio, dry and fresh weights of the seedlings, chlorophyll, carbohydrate and protein contents and enzymatic system in response to heavy metal stress and to determine the effects of heavy metal $\mathrm{Hg}$ on growth and metabolism of crop plants. The findings of present study would not only help in understanding the phyto-toxicity of heavy metal pollution but would also try to suggest the possibilities of selecting suitable varieties of crops for growing best under heavy metal polluted irrigation waters and soils and the control measures to overcome the heavy metal phyto-toxicity.

\section{Material and Methods}

\section{a.Experimental Design}

The four wheat varieties selected for the present investigation are most commonly grown in western U.P. particularly in Mathura and nearby areas. The seeds of the material involved in the present study were kindly provided by the Agriculture Research Centre Raya, Mathura. The following crop plants were chosen for experimentation: The following four wheat varieties (Triticum aestivum L) of the family Poaceae (Gramineae) were selected for experimentation: 

1) Lok1 (V1)
2) UP-2338 (V2)
3) PBW-154 (V3)
4) PBW-502 (V4)

The above crop varieties were screened for heavy metal Hg phyto-toxicity on the following parameters:

1. Germination Percentage (GP)

2. Seed Germination Index (SGI)

Certified seeds of the four local varieties of a cereal (Wheat) crop were screened for their relative tolerance to the Heavy metal mercury as $\mathrm{HgCl}_{2}$ under varying concentration levels viz., 0, 100, 200 and 300ppm. Distilled water was used as control treatments. Observations on seedling growth were recorded as Germination Percentage (GP) Seed Germination Index (SGI) at 24 hours interval from 24 hours after sowing up to the end of 120 hours.

\section{Preparation of Stock Solution:}

Stock solutions for heavy metal mercury as $\mathrm{HgCl}_{2}$ were prepared as follows:

1) $50 \mathrm{ppm}$ stock solution: dissolved $0.05 \mathrm{~g}$ salt in $1000 \mathrm{ml}$ distilled water

2) $100 \mathrm{ppm}$ stock solution: dissolved $0.1 \mathrm{~g}$ salt in $1000 \mathrm{ml}$ distilled water

3) $200 \mathrm{ppm}$ stock solution: dissolved $0.2 \mathrm{~g}$ salt in $1000 \mathrm{ml}$ distilled water

4) $300 \mathrm{ppm}$ stock solution: dissolved $0.3 \mathrm{~g}$ salt in $1000 \mathrm{ml}$ distilled water

\section{Sterilization of seeds in $\mathrm{HgCl} 2$ salt treatment}

Twenty seeds of each variety were surface sterilized by soaking in $0.1 \%(\mathrm{w} / \mathrm{v}) \mathrm{HgCl}_{2}$ for $2 \mathrm{~min}$ and then rinsed twice with sterile distilled water. Sterilized seeds were subjected to heavy metal salt treatment in petri-dishes (for seed germination) containing autoclaved sterilized filter paper (Whatman No1) saturated with different concentrations of $\mathrm{HgCl}_{2}$. Seed germination was calculated after 24, 48, 72, 96 and 120 hours. Three replications were taken for all the experimentation.

\section{Screening of the crops for heavy metal Hg phyto-toxicity:}

Screening of the crops for heavy metal phyto-toxicity was carried out after Garrads Technique [51] as modified by [52] and as per method of [53] and [9,10,54,55] Seed Germination analysis: Germination percentage (GP) at each interval at varying levels of heavy metal $\mathrm{Hg}$ concentration and control (distilled water) was observed along with the speed of germination index (SGI) was determined by following the formula of Carley \& Watson [56]:

$S G I=(5 \times 1 G+4 \times 2 G+3 \times 3 G+2 \times 4 G+1 \times 5 G)$

Where, $1 G---5 G=$ Number of seeds germinated on the first ( 24 hours) to fifth (120 hours) day.

\section{Statistical Analysis}

All parameters with three replicates were analyzed by Analysis of Variance (ANOVA) by using window SPSS 2003. Data were expressed as the mean \pm standard error of the mean. Critical differences at 0.01 and 0.05 per cent probability were calculated wherever the results were significant.

\section{Results}

\section{Germination percentage (GP)}

ANOVA analysis: As indicated in the Table 1 (ANOVA ANALYSISGermination Percentage) all the main effects viz., Variety, Treatment (Heavy metal), Duration and their interactions (A; B; C; A X B; A X C; B X C; A X B X C) were highly significant both at $0.01 \%$ and $0.05 \%$ level of probability as such significant differences were noticed in the germination percentage of the four crop varieties studied Tables 1-10 and Graphs 1-10. Results of all the main effects viz., Variety, Treatment (Heavy metal), Duration and their interactions (A; B; C; A X B; A X C; B X C; A X B X C) are described as follows:

Table 1: Anova table* germination percentage in the four wheat varieties.

\begin{tabular}{|c|c|c|c|c|c|}
\hline Source of variation & DF & SS & MSS & F-value & Significance \\
\hline Factor A (Variety) & 3 & 63.35 & 21.117 & 5.18 & $*, * *$ \\
\hline Factor B (Treatment) & 3 & 85064.58 & 28354.86 & 6965.37 & $*, * *$ \\
\hline Factor C (Duration) & 4 & 72003.11 & 18000.78 & 4421.89 & $*, * *$ \\
\hline Factor A X B & 9 & 250.58 & 27.84 & 6.84 & ), $* *$ \\
\hline Factor A X C & 12 & 224.85 & 18.73 & 4.6 & $*, * *$ \\
\hline Factor B X C & 12 & 6592.79 & 549.39 & 134.96 & $*, * *$ \\
\hline Factor A X B X C & 36 & 444.04 & 12.33 & 3.03 & $*, * *$ \\
\hline Error & 160 & 651.33 & 4.07 & & \\
\hline Total & 239 & 165294.7 & & & \\
\hline
\end{tabular}


Table 2: Effect of heavy metal mercury $\left(\mathrm{Hgcl}_{2}\right)$ on germination percentage of four wheat varieties (variety).

\begin{tabular}{|c|c|c|}
\hline Grand Mean $=\mathbf{3 2 . 9 2 5}$ & \multicolumn{2}{|c|}{ SEM $\pm \mathbf{0 . 1 3}$} \\
\hline Range of Germination & Lower Range $=32.66$ & Upper Range $=33.18$ \\
\hline
\end{tabular}

*Significant at $5 \%$ Level of Probability; ** Significant at $1 \%$ Level of Probability.

\begin{tabular}{|c|c|c|c|}
\hline \multirow{2}{*}{ Variety } & \multirow{2}{*}{ Germination Percentage } & \multicolumn{2}{|c|}{ Range of Germination } \\
\cline { 3 - 4 } & & 33.16 & 34.19 \\
\hline V1 LOK1 & 33.68 & 31.81 & 32.84 \\
\hline V2 UP-2338 & 32.33 & 32.56 & 33.59 \\
\hline V3 PBW-154 & 33.08 & 32.08 & 33.11 \\
\hline V4 PBW-502 & 32 & & \\
\hline SEM \pm 0.26 & & & \\
\hline
\end{tabular}

V1 Lok1 (33.68\%) > V3 PBW 154 (33.08\%) > V2 UP 2338 (32.33\%) > V4 PBW 502 (32.0\%).

Table 3: Effect of heavy metals mercury $\left[\mathrm{Hgcl}_{2}\right]$ on germination percentage of four wheat varieties (treatment).

\begin{tabular}{|c|c|c|c|}
\hline Heavy Metal Mercury $\left(\mathrm{HgCl}_{2}\right)$ & Germination Percentage & \multicolumn{2}{|c|}{ Range of Germination } \\
\cline { 3 - 4 } & & 62.96 & Upper \\
\hline Control DW 0ppm & 63.48 & 30 & 31.03 \\
\hline $100 \mathrm{ppm}$ & 30.51 & 24.73 & 25.76 \\
\hline $200 \mathrm{ppm}$ & 25.25 & 11.93 & 12.96 \\
\hline $300 \mathrm{ppm}$ & 12.45 & & \\
\hline $\mathrm{SEM} \pm 0.26$ & & & \\
\hline
\end{tabular}

Control $(63.48 \%)>100 \mathrm{ppm}(30.51 \%)>200(25.25 \%)>300 \mathrm{ppm}(12.45 \%)$.

Table 4: Effect of heavy metal mercury $\left(\mathrm{Hgcl}_{2}\right)$ on germination percentage of four wheat varieties (duration).

\begin{tabular}{|c|c|c|c|}
\hline \multirow{2}{*}{$\begin{array}{c}\text { Duration (hours) } \\
\end{array}$} & Germination Percentage & Lower & Range of Germination \\
\cline { 3 - 4 } & & 5.84 & 6.99 \\
\hline $24 \mathrm{hrs}$ & 6.41 & 21.5 & 22.65 \\
\hline $48 \mathrm{hrs}$ & 22.08 & 33.69 & 34.84 \\
\hline $72 \mathrm{hrs}$ & 34.27 & 46.48 & 47.63 \\
\hline $96 \mathrm{hrs}$ & 47.06 & 54.21 & 55.36 \\
\hline $120 \mathrm{hrs}$ & 54.79 & & \\
\hline
\end{tabular}

$120 \mathrm{hr}(54.79 \%)>96 \mathrm{hr}(47.06 \%)>72 \mathrm{hr}(34.27 \%)>48 \mathrm{hr}(22.08 \%)>24 \mathrm{hr}(6.41 \%)$.

Table 5: Effect of heavy metal mercury $\left(\mathrm{Hgcl}_{2}\right)$ on germination percentage of four wheat varieties (Variety $\mathrm{x}$ treatment).

\begin{tabular}{|c|c|c|c|c|}
\hline \multirow[t]{2}{*}{ Variety } & \multirow[t]{2}{*}{ Heavy Metal Mercury $\left(\mathrm{HgCl}_{2}\right)$} & \multirow[t]{2}{*}{ Germination Percentage } & \multicolumn{2}{|c|}{ Range of Germination } \\
\hline & & & Lower & Upper \\
\hline \multirow{4}{*}{ V1 LOK1 } & Control DW 0ppm & 62.33 & 61.3 & 63.36 \\
\hline & $100 \mathrm{ppm}$ & 30.46 & 29.43 & 31.49 \\
\hline & $200 \mathrm{ppm}$ & 27.73 & 26.7 & 28.76 \\
\hline & $300 \mathrm{ppm}$ & 14.2 & 13.17 & 15.22 \\
\hline
\end{tabular}




\begin{tabular}{|c|c|c|c|c|}
\hline \multirow[t]{4}{*}{ V2 UP-2338 } & Control DW 0ppm & 62.2 & 61.17 & 63.22 \\
\hline & $100 \mathrm{ppm}$ & 30.93 & 29.9 & 31.96 \\
\hline & $200 \mathrm{ppm}$ & 25 & 23.97 & 26.02 \\
\hline & $300 \mathrm{ppm}$ & 11.2 & 10.17 & 12.22 \\
\hline \multirow[t]{4}{*}{ V3 PBW-154 } & Control DW 0ppm & 65.13 & 64.1 & 66.16 \\
\hline & $100 \mathrm{ppm}$ & 31 & 29.97 & 32.02 \\
\hline & $200 \mathrm{ppm}$ & 24.06 & 23.03 & 25.09 \\
\hline & $300 \mathrm{ppm}$ & 12.13 & 11.1 & 13.16 \\
\hline \multirow[t]{4}{*}{ V4 PBW-502 } & Control DW 0ppm & 64.26 & 63.23 & 65.29 \\
\hline & $100 \mathrm{ppm}$ & 29.66 & 28.63 & 30.69 \\
\hline & $200 \mathrm{ppm}$ & 24.2 & 23.17 & 25.22 \\
\hline & $300 \mathrm{ppm}$ & 12.26 & 11.23 & 13.29 \\
\hline$S E M \pm 0.521$ & & & & \\
\hline
\end{tabular}

V1 LOK1-120hr $(56.417 \%)>96 \mathrm{hr}(47.750 \%)>72 \mathrm{hr}(36.333 \%)>48 \mathrm{hr}(22.000 \%)>24 \mathrm{hr}(5.917 \%)$

V2 UP-2338-20hr (53.167\%) > 96hr (47.833\%) > 72hr $(33.417 \%)>48 \mathrm{hr}(20.750 \%)>24 \mathrm{hr}(6.500 \%)$

V3 PBW-154-120hr (53.583\%) > 96hr (46.833\%) > 72hr (35.000\%) > 48hr (23.417\%) > 24hr (6.583\%)

V4 PBW-502-120hr (56.000\%) > 96hr (45.833\%) > 72hr $(32.333 \%)>48 \mathrm{hr}(22.167 \%)>24 \mathrm{hr}(6.667 \%)$.

Table 6: Effect of heavy metal mercury $\left(\mathrm{Hgcl}_{2}\right)$ on germination percentage of four wheat varieties (variety $\mathrm{x}$ duration).

\begin{tabular}{|c|c|c|c|c|}
\hline \multirow{2}{*}{ Variety } & \multirow{2}{*}{ Duration (hours) } & \multirow{2}{*}{ Germination Percentage } & \multicolumn{2}{|c|}{ Range of Germination } \\
\hline & & & Lower & Upper \\
\hline \multirow[t]{5}{*}{ V1 LOK1 } & $24 \mathrm{hrs}$ & 5.91 & 4.76 & 7.06 \\
\hline & $48 \mathrm{hrs}$ & 22 & 20.85 & 23.15 \\
\hline & $72 \mathrm{hrs}$ & 36.33 & 35.18 & 37.48 \\
\hline & $96 \mathrm{hrs}$ & 47.75 & 46.6 & 48.9 \\
\hline & $120 \mathrm{hrs}$ & 56.41 & 55.26 & 57.56 \\
\hline \multirow{5}{*}{ V2 UP-2338 } & $24 \mathrm{hrs}$ & 6.5 & 5.35 & 7.65 \\
\hline & $48 \mathrm{hrs}$ & 20.7 & 19.6 & 21.9 \\
\hline & $72 \mathrm{hrs}$ & 33.41 & 32.26 & 34.56 \\
\hline & $96 \mathrm{hrs}$ & 47.83 & 46.68 & 48.98 \\
\hline & $120 \mathrm{hrs}$ & 53.16 & 52.01 & 54.31 \\
\hline \multirow[t]{5}{*}{ V3 PBW-154 } & $24 \mathrm{hrs}$ & 6.58 & 5.43 & 7.73 \\
\hline & $48 \mathrm{hrs}$ & 23.41 & 22.26 & 24.56 \\
\hline & $72 \mathrm{hrs}$ & 35 & 33.85 & 36.15 \\
\hline & 96hrs & 46.83 & 45.68 & 47.98 \\
\hline & $120 \mathrm{hrs}$ & 53.58 & 52.43 & 54.73 \\
\hline
\end{tabular}




\begin{tabular}{|c|c|c|c|c|}
\hline \multirow{3}{*}{ V4 PBW-502 } & $24 \mathrm{hrs}$ & 6.66 & 5.51 & 7.81 \\
\cline { 2 - 5 } & $48 \mathrm{hrs}$ & 22.16 & 21.01 & 31.18 \\
\cline { 2 - 5 } & $72 \mathrm{hrs}$ & 32.33 & 44.68 & 33.48 \\
\cline { 2 - 5 } & $96 \mathrm{hrs}$ & 45.83 & 54.85 & 56.98 \\
\cline { 2 - 5 } & \multirow{2}{*}{$120 \mathrm{hrs}$} & 56 & & 57.15 \\
\hline
\end{tabular}

Control-120hr Seedling (91.667) > 96hr Seedling (83.250) > 72hr Seedling (63.667) > 48hr Seedling (53.167) > 24hr Seedling (25.667) 100ppm-120hr Seedling (52.083) > 96hr Seedling (46.167) > 72hr Seedling (34.417) > 48hr Seedling (19.917) > 24hr Seedling (0.000)

200ppm-120hr Seedling (46.083) > 96hr Seedling (40.167) > 72hr Seedling (28.833) > 48hr Seedling (11.167) > 24hr Seedling $(0.000)$

300ppm-120hr Seedling (29.333) > 96hr Seedling (18.667) > 72hr Seedling (10.167) > 48hr Seedling (4.083) > 24hr Seedling $(0.000)$.

Table 7: Effect of heavy metal mercury $\left(\mathrm{Hgcl}_{2}\right)$ on germination percentage of four wheat varieties (duration $\mathrm{x}$ treatment).

\begin{tabular}{|c|c|c|c|c|}
\hline \multirow[t]{2}{*}{ Duration (hours) } & \multirow[t]{2}{*}{ Heavy Metal Mercury $\left(\mathrm{HgCl}_{2}\right)$} & \multirow[t]{2}{*}{ Germination Percentage } & \multicolumn{2}{|c|}{ Range of Germination } \\
\hline & & & Lower & Upper \\
\hline \multirow[t]{4}{*}{$24 \mathrm{hrs}$} & Control DW 0ppm & 25.667 & 24.516 & 26.817 \\
\hline & $100 \mathrm{ppm}$ & 0 & 0 & 0 \\
\hline & 200ppm & 0 & 0 & 0 \\
\hline & 300ppm & 0 & 0 & 0 \\
\hline \multirow[t]{4}{*}{$48 \mathrm{hrs}$} & Control DW 0ppm & 53.167 & 52.016 & 54.317 \\
\hline & $100 \mathrm{ppm}$ & 19.917 & 18.766 & 21.067 \\
\hline & 200ppm & 11.167 & 10.016 & 12.317 \\
\hline & 300ppm & 4.083 & 2.933 & 5.234 \\
\hline \multirow[t]{4}{*}{$72 \mathrm{hrs}$} & Control DW 0ppm & 63.667 & 62.516 & 64.817 \\
\hline & $100 \mathrm{ppm}$ & 34.417 & 33.266 & 35.567 \\
\hline & 200ppm & 28.833 & 27.683 & 29.984 \\
\hline & 300ppm & 10.167 & 9.016 & 11.317 \\
\hline \multirow[t]{4}{*}{$96 \mathrm{hrs}$} & Control DW 0ppm & 83.25 & 82.1 & 84.4 \\
\hline & $100 \mathrm{ppm}$ & 46.167 & 45.016 & 47.317 \\
\hline & 200ppm & 40.167 & 39.016 & 41.317 \\
\hline & 300ppm & 18.667 & 17.516 & 19.817 \\
\hline \multirow{4}{*}{$120 \mathrm{hrs}$} & Control DW 0ppm & 91.667 & 90.516 & 92.817 \\
\hline & $100 \mathrm{ppm}$ & 52.083 & 50.933 & 53.234 \\
\hline & 200ppm & 46.083 & 44.933 & 47.234 \\
\hline & $300 \mathrm{ppm}$ & 29.333 & 28.183 & 30.484 \\
\hline $\mathrm{SEM} \pm 0.582$ & & & & \\
\hline
\end{tabular}


Table 8: Effect of different levels of heavy metal mercury $(\mathrm{Hg})$ exposure on germination percentage in the four wheat varieties (variety $\mathrm{x}$ duration $\mathrm{x}$ treatment).

\begin{tabular}{|c|c|c|c|c|c|}
\hline \multirow{2}{*}{ Variety } & \multirow{2}{*}{ Duration (hours) } & \multicolumn{4}{|c|}{ Treatment $\mathrm{HgCl}_{2}$ Conc. (ppm) } \\
\hline & & Control DW 0ppm & 100ppm & 200ppm & 300ppm \\
\hline \multirow[t]{5}{*}{ V1 LOK-1 } & $24 \mathrm{hrs}$ & 23.66 & 0 & 0 & 0 \\
\hline & $48 \mathrm{hrs}$ & 51 & 21 & 11 & 5 \\
\hline & $72 \mathrm{hrs}$ & 61.33 & 35 & 37 & 12 \\
\hline & $96 \mathrm{hrs}$ & 82.33 & 44 & 43 & 21.66 \\
\hline & $120 \mathrm{hrs}$ & 93.33 & 52.3 & 47.66 & 32.33 \\
\hline \multirow[t]{5}{*}{ V2 UP-2338 } & $24 \mathrm{hrs}$ & 26 & 0 & 0 & 0 \\
\hline & $48 \mathrm{hrs}$ & 52.66 & 16 & 10.33 & 4 \\
\hline & $72 \mathrm{hrs}$ & 63.66 & 36 & 26 & 8 \\
\hline & 96hrs & 82 & 49 & 42.33 & 18 \\
\hline & $120 \mathrm{hrs}$ & 86.66 & 53.7 & 46.33 & 26 \\
\hline \multirow[t]{5}{*}{ V3 PBW-154 } & $24 \mathrm{hrs}$ & 26.33 & 0 & 0 & 0 \\
\hline & $48 \mathrm{hrs}$ & 54.33 & 21.3 & 13.66 & 4.33 \\
\hline & $72 \mathrm{hrs}$ & 66.66 & 35.3 & 26.66 & 11.33 \\
\hline & $96 \mathrm{hrs}$ & 84.33 & 47 & 38 & 18 \\
\hline & $120 \mathrm{hrs}$ & 94 & 51.3 & 42 & 27 \\
\hline \multirow[t]{5}{*}{ V4 PBW-502 } & $24 \mathrm{hrs}$ & 26.66 & 0 & 0 & 0 \\
\hline & $48 \mathrm{hrs}$ & 54.66 & 21.3 & 9.66 & 3 \\
\hline & $72 \mathrm{hrs}$ & 63 & 31.3 & 25.66 & 9.33 \\
\hline & 96hrs & 84.33 & 44.7 & 37.33 & 17 \\
\hline & $120 \mathrm{hrs}$ & 92.66 & 51 & 48.33 & 32 \\
\hline $\mathrm{SEM} \pm 1.16$ & & & & & \\
\hline
\end{tabular}

Table 9: Effect of different levels of heavy metal mercury (hg) exposure on germination percentage in the four wheat varieties $\mathrm{v} x \mathrm{~d} x \mathrm{t}$ (percent over control).

\begin{tabular}{|c|c|c|c|c|c|}
\hline \multirow[t]{2}{*}{ Variety } & \multirow[t]{2}{*}{ Duration (hours) } & \multicolumn{4}{|c|}{ Treatment $\mathrm{HgCl}_{2}$ Conc. (ppm) } \\
\hline & & Control DW 0ppm & 100ppm & 200ppm & $300 \mathrm{ppm}$ \\
\hline \multirow[t]{5}{*}{ V1 LOK-1 } & $24 \mathrm{hrs}$ & 100 & 0 & 0 & 0 \\
\hline & $48 \mathrm{hrs}$ & 100 & 41.2 & 21.56 & 9.8 \\
\hline & $72 \mathrm{hrs}$ & 100 & 57.1 & 60.32 & 19.56 \\
\hline & $96 \mathrm{hrs}$ & 100 & 53.4 & 52.22 & 26.31 \\
\hline & $120 \mathrm{hrs}$ & 100 & 56.1 & 51.07 & 34.64 \\
\hline \multirow[t]{5}{*}{ V2 UP-2338 } & $24 \mathrm{hrs}$ & 100 & 0 & 0 & 0 \\
\hline & $48 \mathrm{hrs}$ & 100 & 30.4 & 19.61 & 7.59 \\
\hline & $72 \mathrm{hrs}$ & 100 & 56.5 & 40.83 & 12.56 \\
\hline & 96hrs & 100 & 59.8 & 51.62 & 21.95 \\
\hline & $120 \mathrm{hrs}$ & 100 & 61.9 & 53.46 & 29.99 \\
\hline
\end{tabular}




\begin{tabular}{|c|c|c|c|c|c|}
\hline \multirow{5}{*}{ V3 PBW-154 } & $24 \mathrm{hrs}$ & 100 & 0 & 0 & 0 \\
\hline & $48 \mathrm{hrs}$ & 100 & 39.3 & 25.15 & 7.97 \\
\hline & $72 \mathrm{hrs}$ & 100 & 53 & 40 & 16.99 \\
\hline & $96 \mathrm{hrs}$ & 100 & 55.7 & 45.05 & 21.34 \\
\hline & $120 \mathrm{hrs}$ & 100 & 54.6 & 44.68 & 28.72 \\
\hline \multirow{5}{*}{ V4 PBW-502 } & $24 \mathrm{hrs}$ & 100 & 0 & 0 & 0 \\
\hline & $48 \mathrm{hrs}$ & 100 & 39 & 17.68 & 5.48 \\
\hline & $72 \mathrm{hrs}$ & 100 & 49.7 & 40.74 & 14.81 \\
\hline & $96 \mathrm{hrs}$ & 100 & 53 & 44.26 & 20.15 \\
\hline & $120 \mathrm{hrs}$ & 100 & 55 & 52.15 & 34.53 \\
\hline $\mathrm{SEM} \pm 16$ & & & & & \\
\hline
\end{tabular}

Table 10: Effect of different levels of heavy metal mercury (hg) exposure on germination percentage in the four wheat varieties (percent over control) (data recorded after 72 hours of presoaking the seeds in test solutions).

\begin{tabular}{|c|c|c|c|c|}
\hline \multirow[t]{2}{*}{ Variety } & \multicolumn{4}{|c|}{ Treatment $\mathrm{HgCl}_{2}$ Conc. (ppm) } \\
\hline & Control DW ppm & 100ppm & 200ppm & 300ppm \\
\hline V1 LOK-1 & 100 & 57.06 & 60.3 & 19.56 \\
\hline V2 UP-2338 & 100 & 56.54 & 40.8 & 12.56 \\
\hline V3 PBW-154 & 100 & 52.99 & 40 & 16.99 \\
\hline V4 PBW-502 & 100 & 49.73 & 40.7 & 14.81 \\
\hline $\mathrm{SEM} \pm 1.16$ & & & & \\
\hline
\end{tabular}

V1 LOK1-Control $(100 \%)>200(60.326 \%)>100 \mathrm{ppm}(57.065 \%)>300 \mathrm{ppm}(19.565 \%)$

V2 UP-2338-Control $(100 \%)>100 \mathrm{ppm}(56.544 \%)>200(40.837 \%)>300 \mathrm{ppm}(12.565 \%)$

V3 PBW-154-Control $(100 \%)>100 p p m(52.999 \%)>200(40.0007 \%)>300 p p m(16.999 \%)$

V4 PBW-502-Control $(100 \%)>100 \mathrm{ppm}(49.734 \%)>200(40.741 \%)>300 \mathrm{ppm}(14.814 \%)$

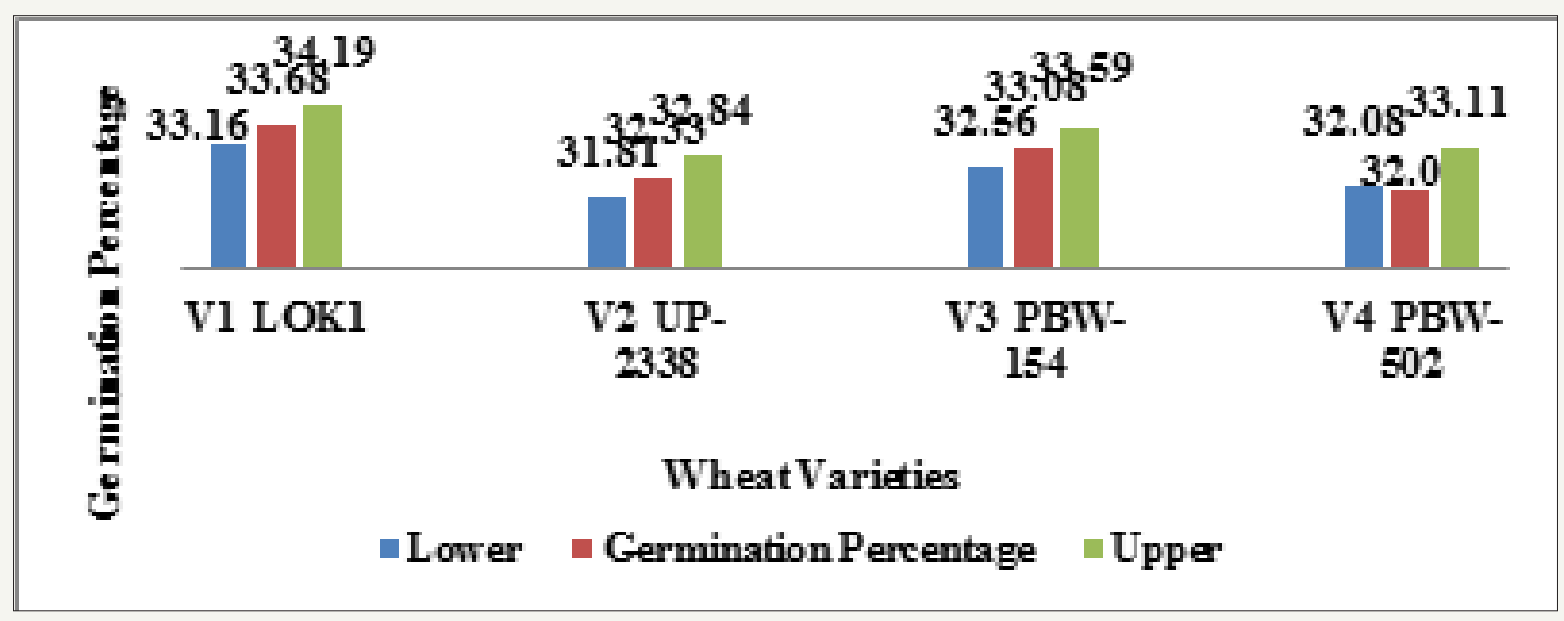

Graph 1: Effect of heavy metal mercury [Hgcl2] on germination percentage of four wheat varieties (variety). 


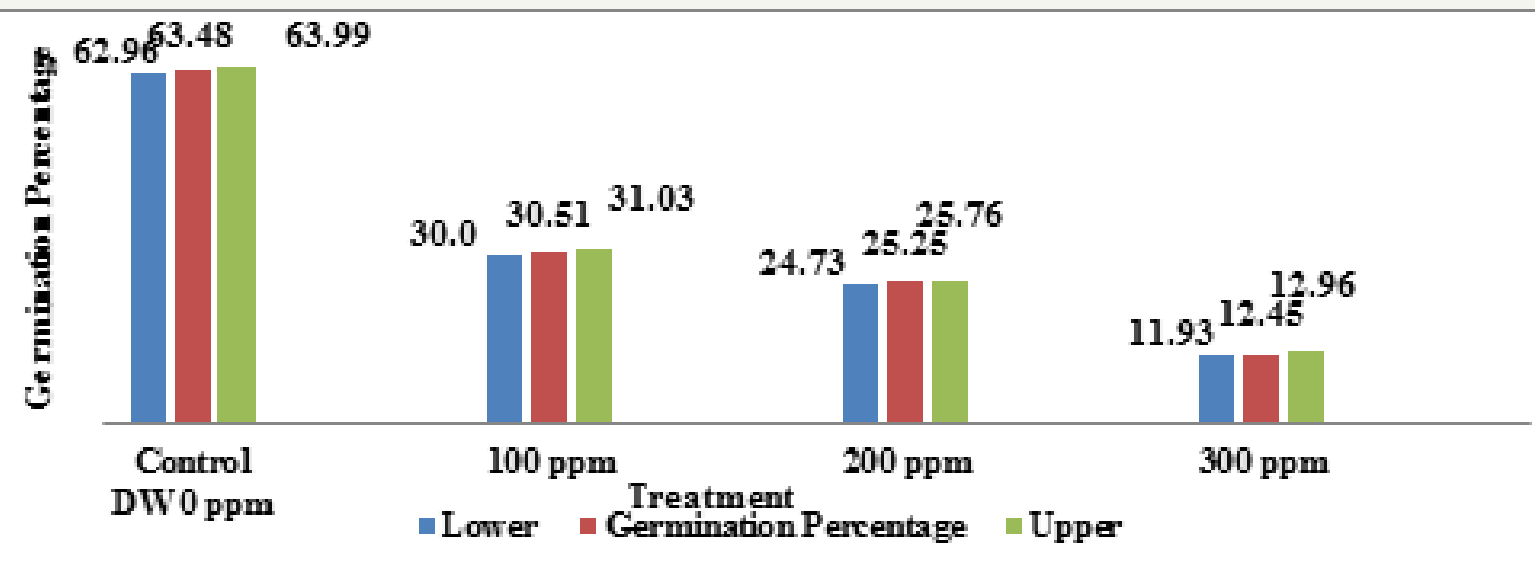

Graph 2: Effect of heavy metals mercury $\left[\mathrm{Hgcl}_{2}\right]$ on germination percentage of four wheat varieties (Treatment).

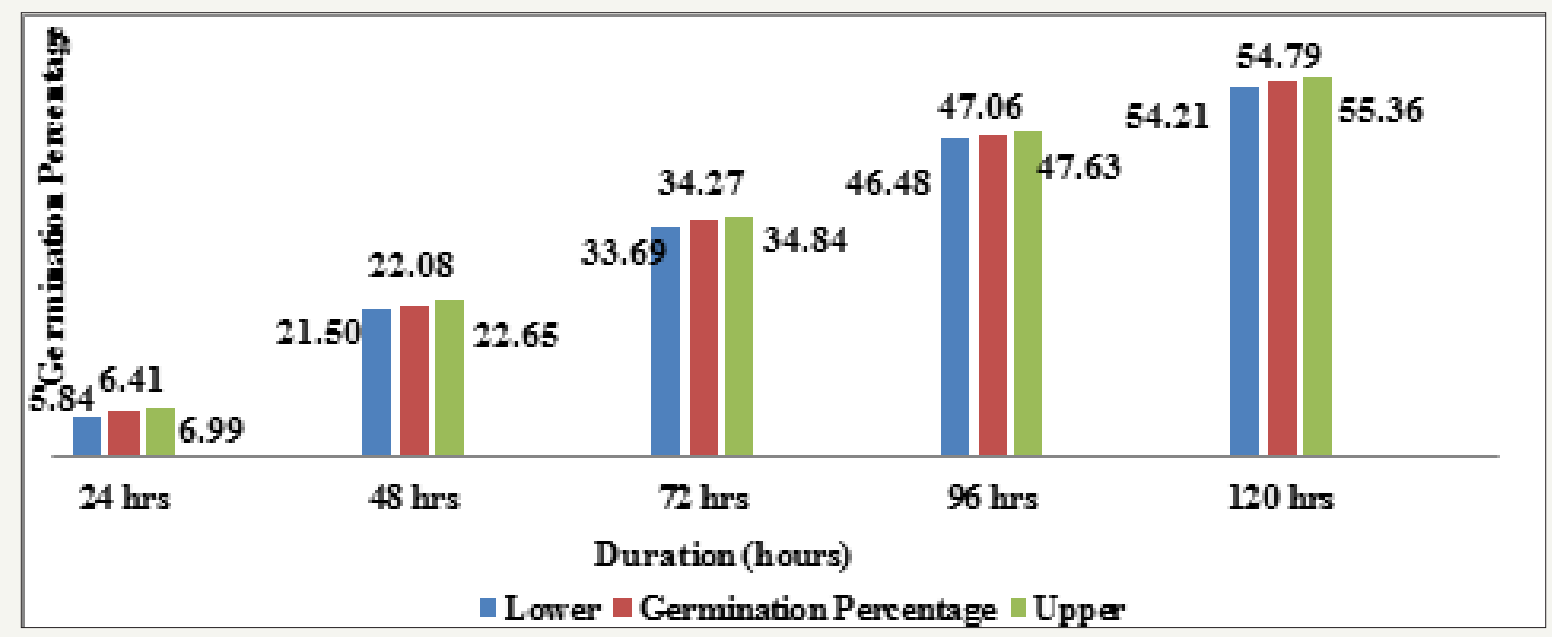

Graph 3: Effect of heavy metals mercury $\left[\mathrm{Hgcl}_{2}\right]$ on germination percentage of four wheat varieties (Duration).

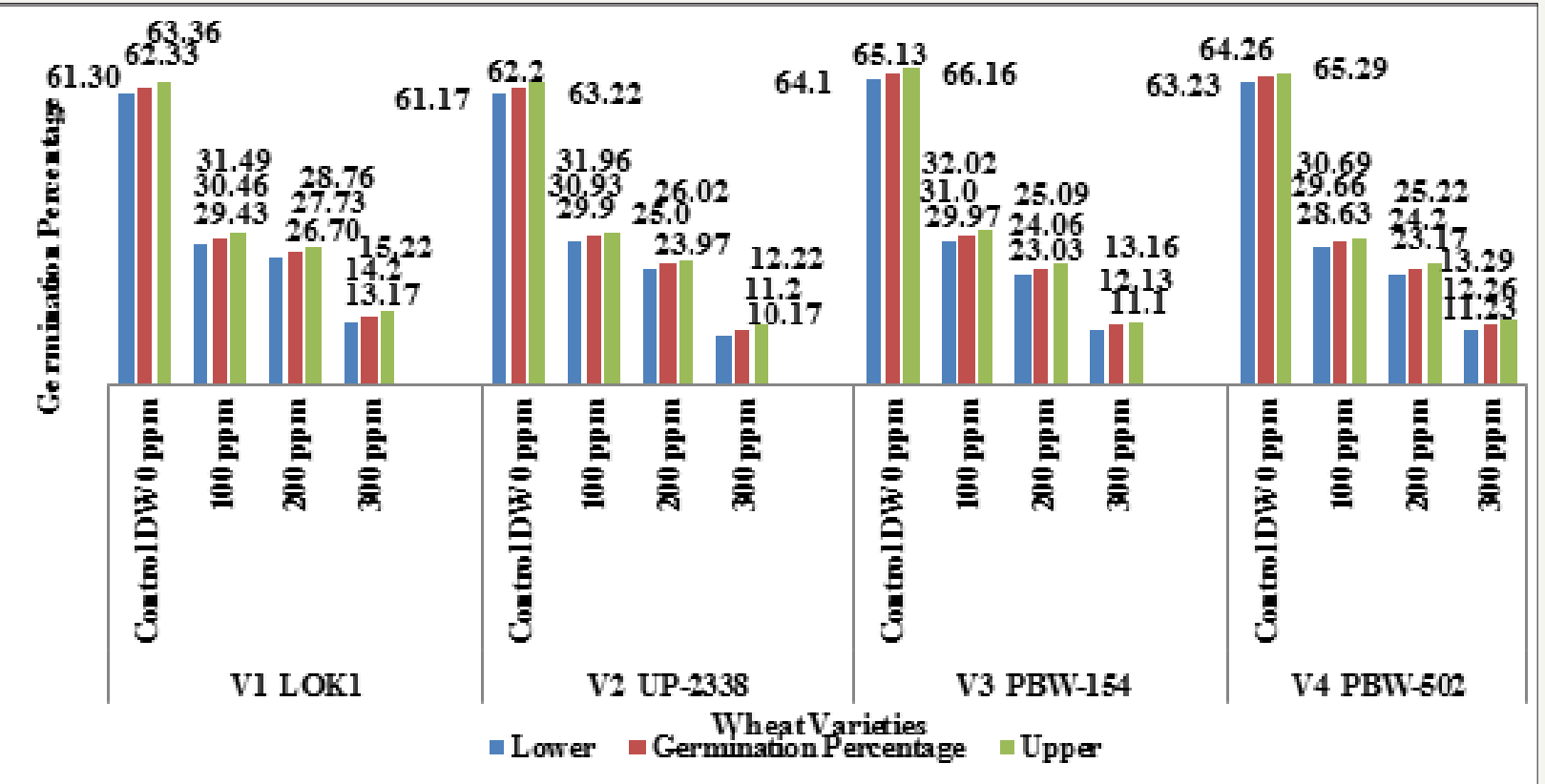

Graph 4: Effect of heavy metal mercury (Hg) on germination percentage of four wheat varieties (Variety $\mathrm{X}$ treatment). 


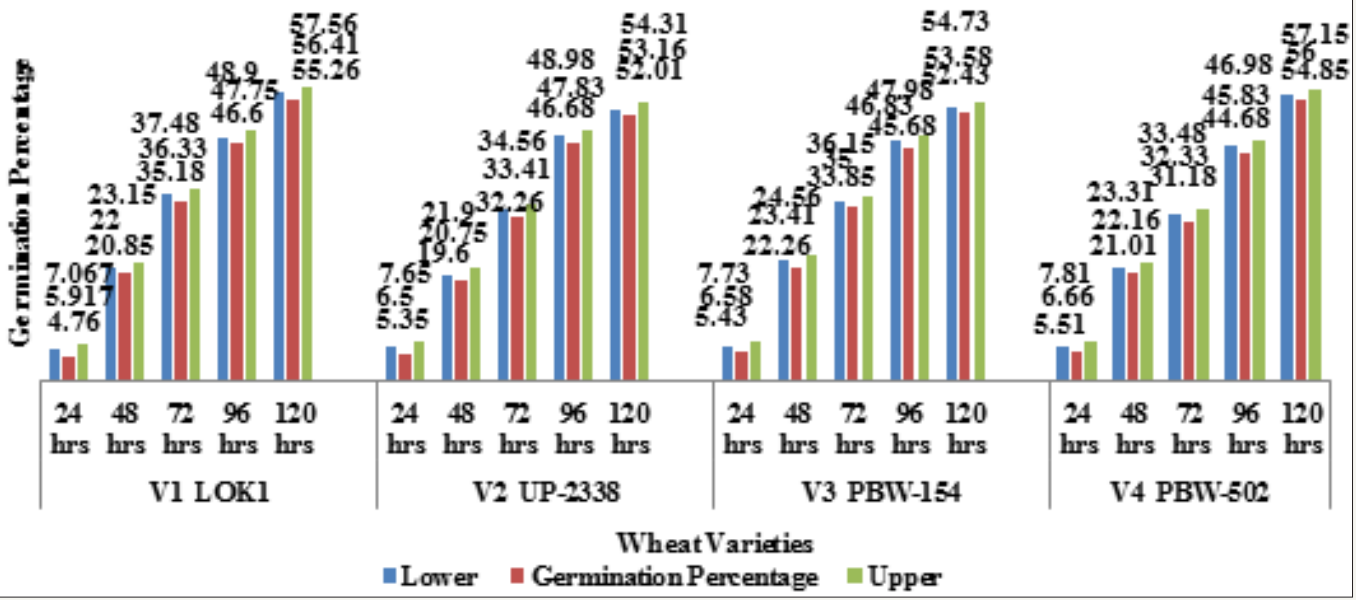

Graph 5: Effect of heavy metal mercury $\left[\mathrm{hgcl}_{2}\right]$ on germination percentage of four wheat varieties (variety $\mathrm{x}$ duration).

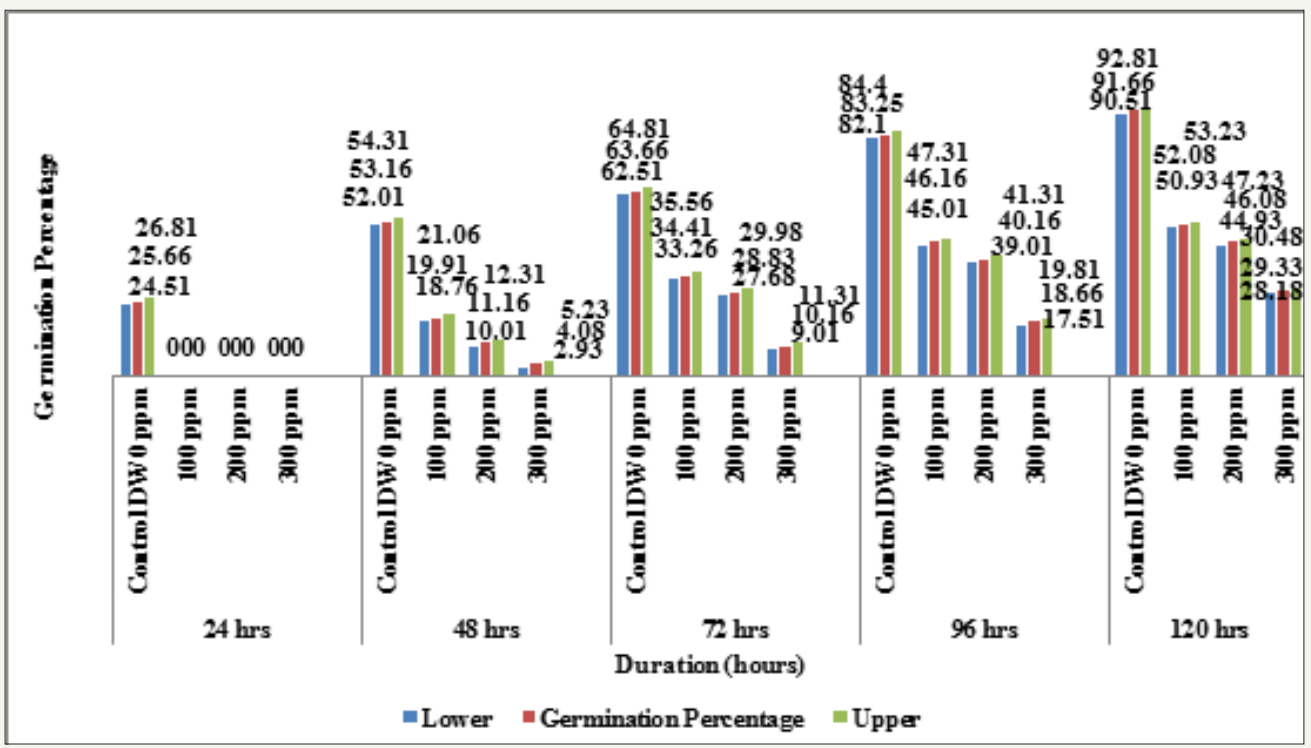

Graph 6: Effect of heavy metal mercury $\left[\mathrm{hgcl}_{2}\right]$ on germination percentage of four wheat varieties (duration $\mathrm{x}$ treatment).

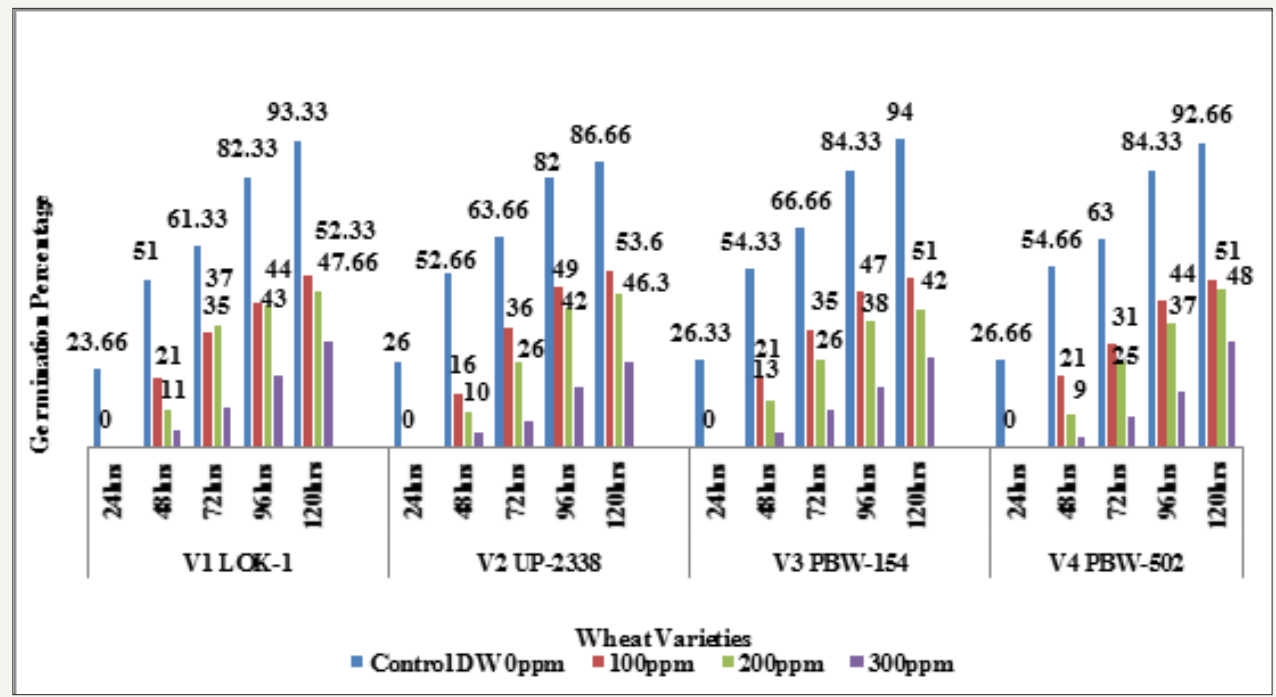

Graph 7: Effect of different levels of heavy metal mercury (hg) exposure on germination percentage in the four wheat varieties (variety $\mathrm{x}$ duration $\mathrm{x}$ treatment). 


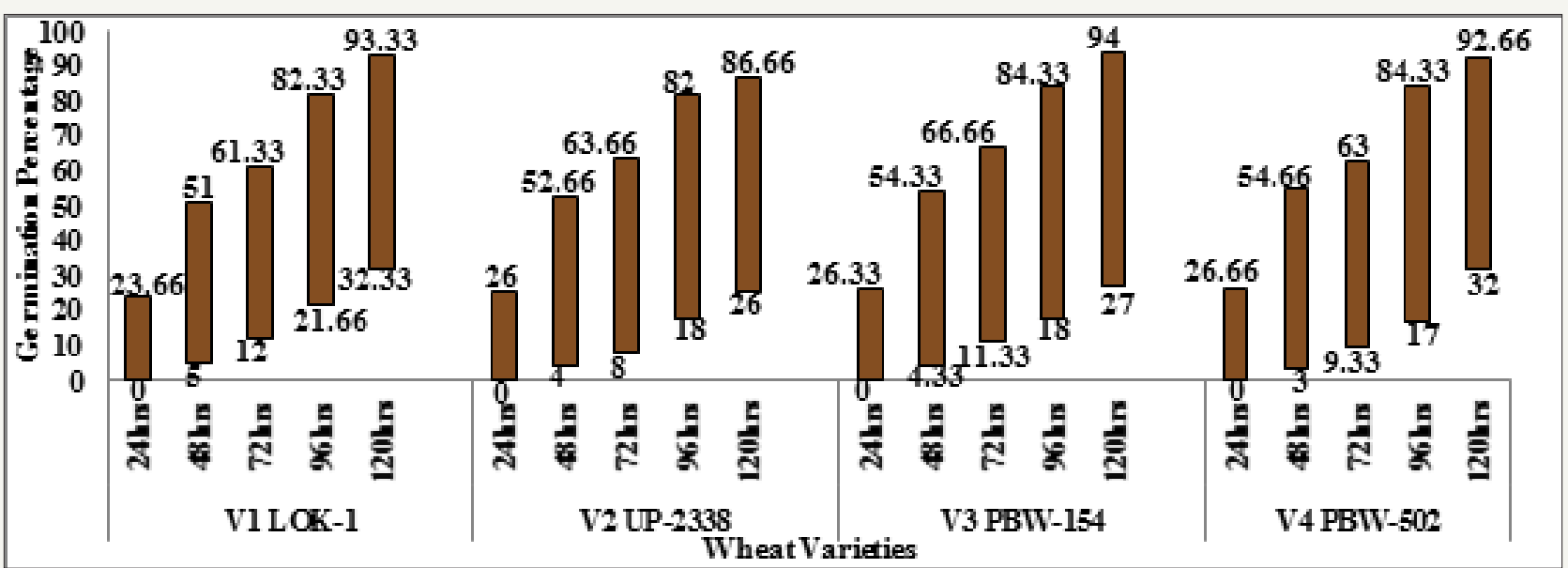

Graph 8: Effect of different levels of heavy metal mercury (hg) exposure on germination percentage in the four wheat varieties (variety $\mathrm{x}$ duration $\mathrm{x}$ treatment).

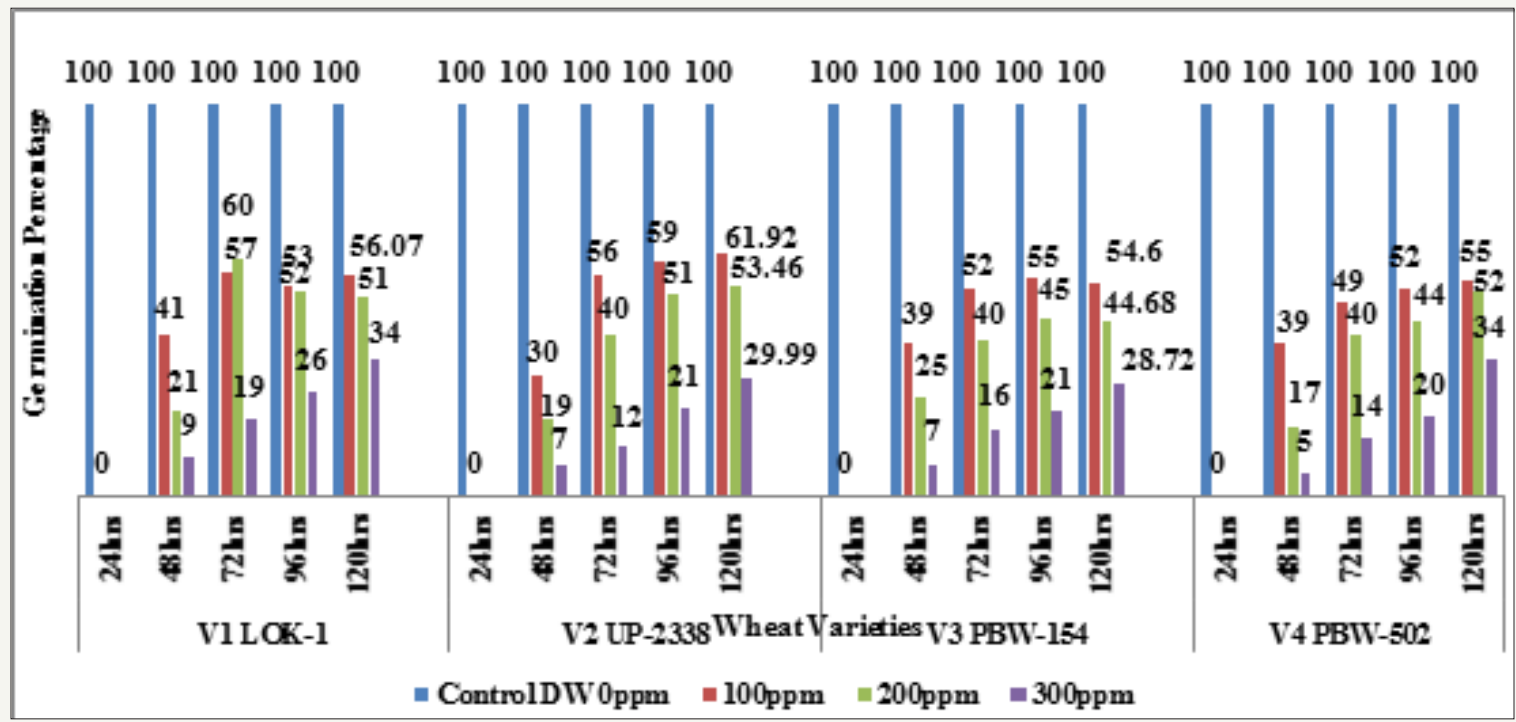

Graph 9: Effect of different levels of heavy metal mercury (hg) exposure on germination percentage in the four wheat varieties (percent over control).

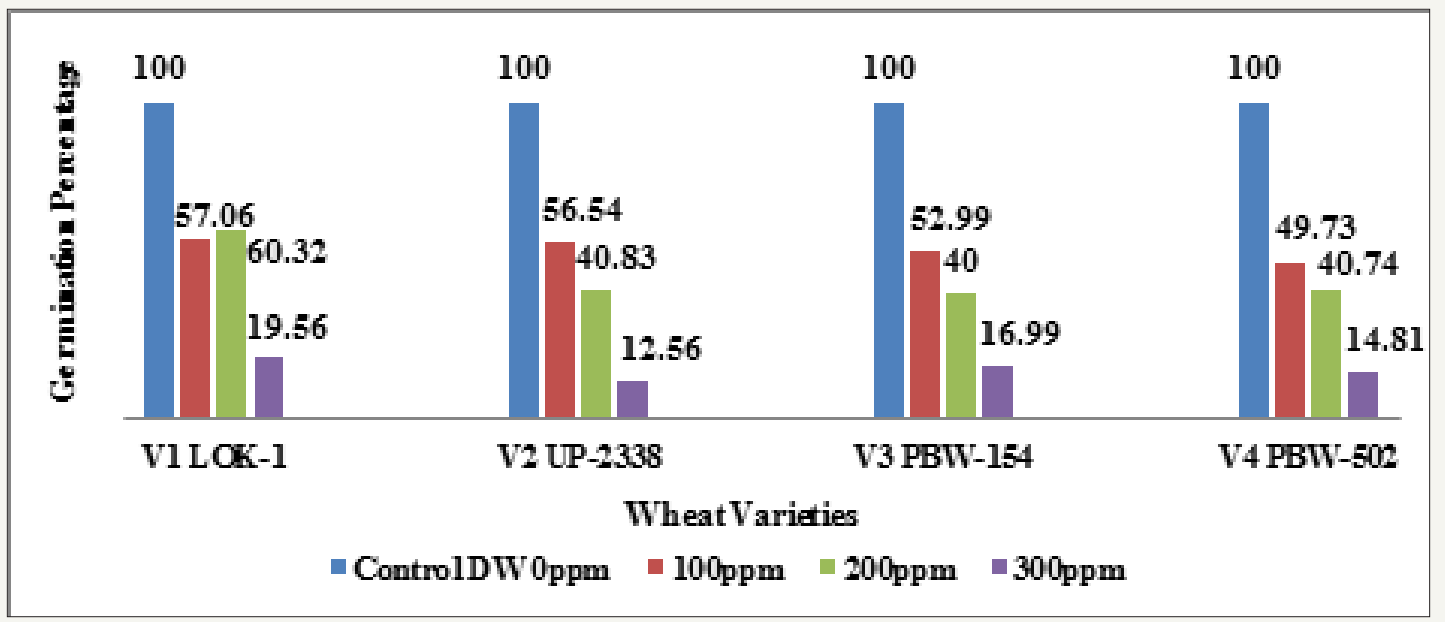

Graph 10: Effect of different levels of heavy metal mercury (hg) exposure on germination percentage in the four wheat varieties (percent over control)(data recorded after 72 hours of presoaking the seeds in test solutions). 
Main effect variety: The highest mean germination percentage $(33.68 \%)$ was recorded in the variety V1 followed by V3, V2 and lastly V4 with the lowest germination percentage (32.0\%). The arieties were arranged in the following descending order on the basis of their respective germination percentage irrespective of the treatment and seedling age (Variety) (Table 2 \& Graph 1). A significant reduction in germination percentage with increasing heavy metal levels was observed irrespective of variety, salt and seedling age (Treatment). The reduction in germination percentage was more pronounced after 200ppm of Hg treatment. Further, with $\mathrm{Hg}$ treatment germination percentage was highest in controls (63.48\%) while lowest (12.45\%) with 300ppm (Table 3 \& Graph 2 ) and the treatments were arranged in the following descending order. The significant interaction of varieties with treatment (Variety X Treatment) is depicted in Tables 4 \& 5 and Graph 4. All the four varieties showed a decrease in germination percentage with increasing salt treatment however, the genotypic variations were quite evident and were arranged in the following descending order on the basis of their respective germination percentage irrespective of the treatment.

All the crop varieties showed an increase in germination percentage (interaction Variety X Duration) exhibiting marked differences in their early seedling growth with advancement in seedling age and the effect of salt declined, i.e., in general, tolerance to heavy metal increased (Table $6 \&$ Graph 5) and were arranged in the following descending order on the basis of their respective germination percentage irrespective of the treatment

Interaction duration $\mathbf{X}$ treatment: The interaction of Duration $X$ Treatment (Table 7 \& Graph 6) shows that with increasing salt concentration level the deleterious salt effect was clearly observable which, however, declined with seedling age. Initially at 24 hours seedling age control sets showed $25.667 \%$ germination but no germination was observed in the Hg treated sets of 100 , 200 and $300 \mathrm{ppm}$. The treatments were arranged in the following descending order

Interaction Variety $\mathbf{X}$ Duration $\mathbf{X}$ Treatment: The results in relation to the effect of different concentrations of $\mathrm{HgCl}_{2}$ on germination performance measured in terms of percent germination after $24,48,72,96$ and 120 hours of sowing have been shown in Tables 1-10 and Graphs 1-10. The germination percentage was significantly inhibited by $\mathrm{Hg}$ in all the four wheat varieties. The degree of inhibition varied depending on the concentration of heavy metal Hg. A review of the final interaction (Variety X Duration $\mathrm{X}$ Treatment) reveals that irrespective of salt concentration levels the germination percentage had increased with seedling age and that the salt treatments had their individual effect depending upon the varying treatment levels (Table 8 and Graph 7 \& 8)

The germination decreased with increasing concentration of mercury in the four wheat varieties studied viz., V1 Lok1, V2 UP2338, V3 PBW-154 and V4 PBW-502 (Tables 1-10 \& Graphs 1-10). A comparison with controls in the four varieties studied showed in the controls: at 24 hours lowest in the variety V1 (23.66\%) and highest in V4 (26.66\%); at 48 hours lowest in V1 (51.0\%) and highest in V4 (54.66\%); at 72 hours lowest in V1 (61.33\%) and highest in V3
(66.66\%); at 96 hours lowest in V2 (82.0\%) and highest in V3 and V4 (84.33\%) and at 120 hours lowest in V2 (86.0\%) and highest in V3 (94.0\%) respectively, whereas in 100, 200 and 300ppm of mercury there was no germination found at all at 24 hours in all the four varieties studied; in 100ppm at 48 hours lowest in V2 (16.0\%) and highest in V3 and V4 (21.33\%); at 72 hours lowest in V4 (31.33\%) and highest in V2 (36.0\%); at 96 hours lowest in V1 (44.0\%) and highest in V2 (49.0\%) and at 120 hours lowest in V4 (51.0\%) and highest in V2 (53.66\%) respectively; at 200ppm at 48 hours lowest in V4 (09.66\%) and highest in V3 (13.66\%) respectively; at 72 hours lowest in V4 (25.66\%) and highest in V1 (37.0\%); 96 hours lowest in V4 (37.33\%) and highest in V1 (43.0\%) and at 120 hours lowest in V3 (42.0\%) and highest in V4 (48.33\%) respectively and at $300 \mathrm{ppm}$ at 48 hours lowest in V4 (03.0\%) and highest in V1 (5.0\%); at 72 hours lowest in V2 (08.0\%) and highest in V1 (12.0\%); 96 hours lowest in V4 (17.0\%) and highest in V1 $(21.66 \%)$ and at 120 hours lowest in V2 (26.0\%) and highest in V1 (32.33\%) respectively (Table 8 and Graph 7 \& 8).

Seeing overall results, it was recorded that the lowest germination percent $(3.0 \%)$ was found in $300 \mathrm{ppm}$ of $\mathrm{Hg}$ solution at 48 hours of germination in the variety V4 whereas highest (94.0\%) was recorded in Controls at 120 hours in the variety V3. Thus, it has been observed that the varieties V1 and V4 behaved better, even in $300 \mathrm{ppm}$ of $\mathrm{Hg}$ concentration at 120 hours of seedling growth. Thus, varieties show overall germination $\%$ as $-\mathrm{V} 1>\mathrm{V} 4>\mathrm{V} 3>\mathrm{V} 2$. Germination percentage as percent over control showed lowest rate $(5.48 \%)$ at 48 hours in 300ppm in the Variety V4 and the highest of $(61.92 \%)$ at 120 hours in 100 ppm Hg in the variety V2 (Table $9 \&$ Graph 9). On the basis of percent over control varieties are placed as $-\mathrm{V} 1>\mathrm{V} 4>\mathrm{V} 2>\mathrm{V} 3$. Data recorded after 72 hours of presoaking the seeds in test solution have shown highest rate of germination $(60.32 \%)$ in $200 p p m$ in the variety $\mathrm{V} 1$ and lowest $(12.56 \%)$ in $300 \mathrm{ppm}$ in the variety $\mathrm{V} 2$ and the overall better performance of the variety V1 in all the treatments (V1>V3>V4>V2) (Table 10 \& Graph $10)$ and were arranged in the following descending order:

\section{Speed of germination index (SGI)}

The results for Speed of Germination Index (SGI) have been depicted in the Tables 11-13 \& Graphs 11-13. The four wheat varieties at 100, 200 and $300 \mathrm{ppm} \mathrm{Hg}$ treatment concentrations showed a gradual decrease in SGI from highest (811.66) in the Var. V3 PBW-154 in the Control Sets while lowest (102.0) was recorded in the Var. V2 UP-2338 in the treatment sets of 300ppm. When SGI was calculated under varying concentration levels of heavy metal Hg Treatment as percent over Control, the lowest SGI was recorded in the Var. V2 UP-2338 (13.03) at 300ppm whereas highest was in the Var. V1 LOK-1 (43.08). The decrease (-) in SGI of treated seeds over control minimum decrease $(-56.91)$ was found in the Var. V1 LOK-1 at 100ppm and maximum decrease (-86.96) was in the Var. V2 UP-2338 at 300ppm heavy metal conc. followed by Var. V4 PBW502 (-86.78), V3 PBW-154 (-85.91) and V1 LOK-1 (-82.77) also in $300 \mathrm{ppm}$. Compared to SGI, GP shown as percent over Control had recorded lowest of $18.0 \%$ in the Var. V2 UP-2338 at $300 \mathrm{ppm}$ whereas the highest GP of $48.98 \%$ was also found in the same variety at $100 \mathrm{ppm}$ (Table $12 \&$ Graph 11$)$. 


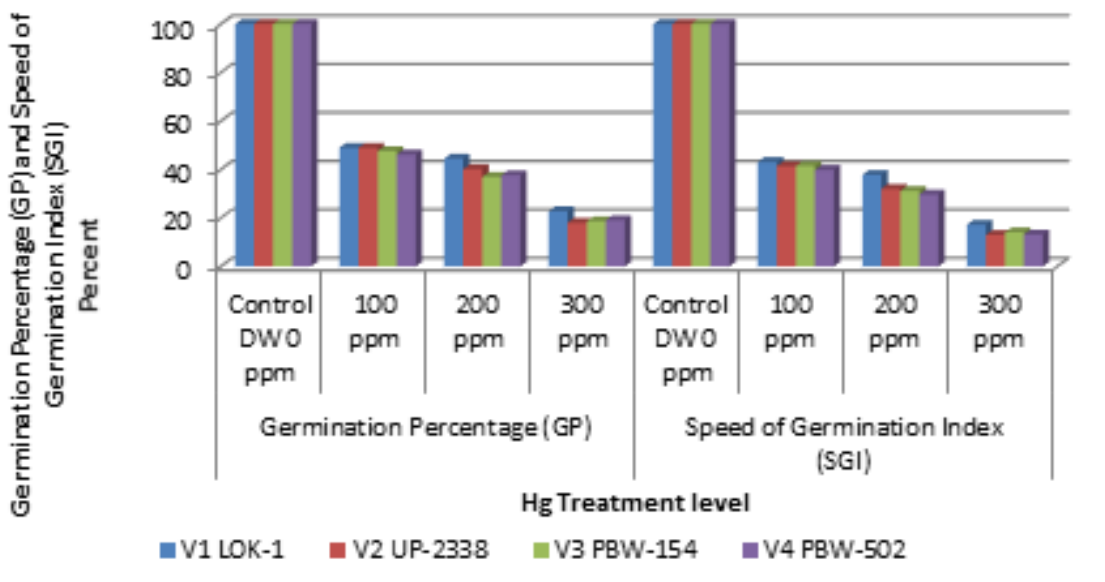

Graph 11: Effect of varying heavy metal mercury concentration on germination percentage (gp) and speed of germination index (sgi) in the four crops (percent over control).

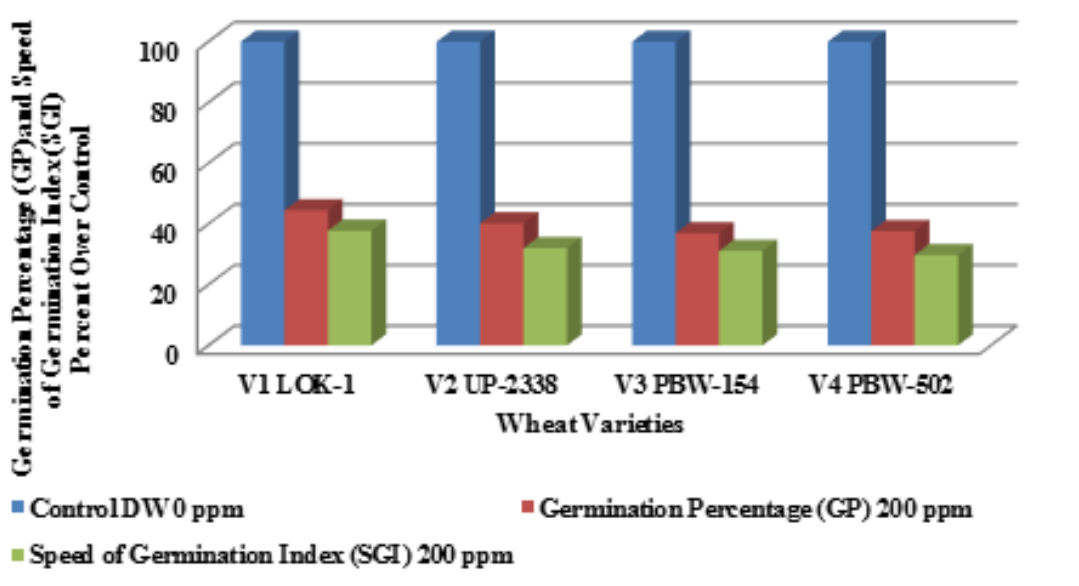

Graph 12: Effect of varying heavy metal mercury concentration on germination percentage (gp) and speed of germination index (sgi) in the four crops [percentover control] at critical level of 200ppm.

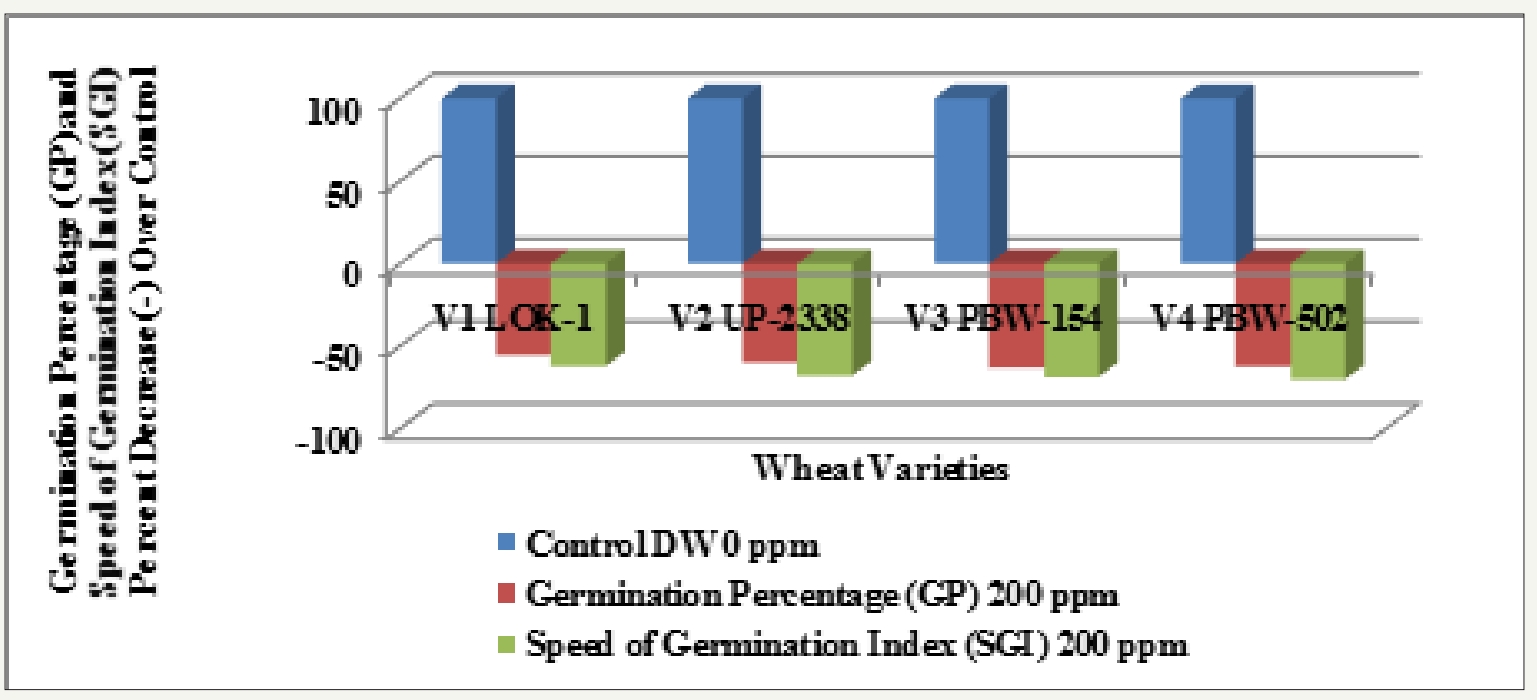

Graph 13: Effect of varying heavy metal mercury concentration on germination percentage (gp) and speed of germination index (sgi) in the four crops [percent decrease (-) over control] at critical level of $200 \mathrm{ppm}$. 
Table 11: Effect of varying heavy metal mercury concentration on speed of germination index (sgi) in the four crops.

\begin{tabular}{|c|c|c|c|c|}
\hline \multirow{2}{*}{ Wheat Varieties } & \multicolumn{4}{|c|}{ Speed of Germination Index (SGI) } \\
\cline { 2 - 5 } & \multicolumn{3}{|c|}{ Treatment HgCl $_{2}$ Conc. (ppm) } \\
\cline { 2 - 5 } & Control DW 0ppm & $\mathbf{1 0 0 p p m}$ & $\mathbf{2 0 0 p p m}$ & $\mathbf{3 0 0 p p m}$ \\
\hline V1 LOK-1 & 764.33 & 329.33 & 288.66 & 131.66 \\
\hline V2 UP-2338 & 782.33 & 323.66 & 250.33 & 102 \\
\hline V3 PBW-154 & 811.66 & 336.66 & 252.66 & 114.33 \\
\hline V4 PBW-502 & 802.33 & 319.66 & 238.66 & 105.99 \\
\hline
\end{tabular}

Table 12: Effect of varying heavy metal mercury concentration on germination percentage (gp) and speed of germination index (sgi) in the four crops (percent over control).

\begin{tabular}{|c|c|c|c|c|c|c|c|c|}
\hline \multirow{2}{*}{$\begin{array}{c}\text { Wheat Vari- } \\
\text { eties }\end{array}$} & \multicolumn{3}{|c|}{$\begin{array}{c}\text { Germination Percentage (GP) Per cent Over } \\
\text { Control }\end{array}$} & \multicolumn{5}{c|}{ Speed of Germination Index (SGI) Per cent Over Control } \\
\cline { 2 - 9 } & $\begin{array}{c}\text { Control DW } \\
\text { 0ppm }\end{array}$ & $\mathbf{1 0 0 p p m}$ & $\mathbf{2 0 0 p p m}$ & $\mathbf{3 0 0 p p m}$ & $\begin{array}{c}\text { Control DW } \\
\text { 0ppm }\end{array}$ & $\mathbf{1 0 0 p p m}$ & $\mathbf{2 0 0 p p m}$ & $\mathbf{3 0 0 p p m}$ \\
\hline \multirow{2}{*}{ V1 LOK-1 } & 100 & 48.97 & 44.49 & 22.78 & 100 & 43.08 & 37.76 & 17.22 \\
\cline { 2 - 9 } & & $*(-51.02)$ & $*(-55.5)$ & $*(-77.22)$ & & $*(-56.91)$ & $*(-62.23)$ & $*(-82.77)$ \\
\hline V2 UP-2338 & 100 & 48.98 & 40.19 & 18 & 100 & 41.37 & 31.99 & 13.03 \\
\cline { 2 - 9 } & & $*(-51.02)$ & $*(-59.8)$ & $*(-81.99)$ & & $*(-58.62)$ & $*(-68.00)$ & $*(-86.96)$ \\
\hline V3 PBW-154 & 100 & 47.59 & 36.94 & 18.62 & 100 & 41.47 & 31.12 & 14.08 \\
\cline { 2 - 9 } & & $*(-52.40)$ & $*(-63.05)$ & $*(-81.37)$ & & $*(-58.52)$ & $*(-68.87)$ & $*(-85.91)$ \\
\hline V4 PBW-502 & 100 & 46.16 & 37.65 & 19.08 & 100 & 39.84 & 29.74 & 13.21 \\
\cline { 3 - 9 } & & $*(-53.83)$ & $*(-62.34)$ & $*(-80.91)$ & & $*(-60.15)$ & $*(-70.25)$ & $*(-86.78)$ \\
\hline
\end{tabular}

*Values in Parenthesis show Percent Decrease (-) over Control in GP and SGI

Percent over control

GP: V1 LOK-1 (44.49\%)> V2 UP-2338 (40.19\%)> V4 PBW-502 (37.65\%)> V3 PBW-154 (36.94\%)

SGI: V1 LOK-1 (37.76\%)> V2 UP-2338 (31.99\%)> V3 PBW-154 (31.12\%)> V4 PBW-502 (29.74\%)

Percent decrease (-) over control

GP: V1 LOK-1 (-55.5\%)< V2 UP-2338 (-59.8\%)< V4 PBW-502 (-62.34\%)< V3 PBW-154 (-63.05\%)

SGI: V1 LOK-1 (-62.23\%)< V2 UP-2338 (-68.0\%)< V3 PBW-154 (-68.87\%)< V4 PBW-502 (-70.25\%).

Table 13: Effect of varying heavy metal mercury concentration on germination percentage (gp) and speed of germination index (sgi) in the four crops [percent decrease (-) over control] at critical level of 200ppm.

\begin{tabular}{|c|c|c|c|}
\hline \multirow{2}{*}{ Wheat Varieties } & Control DW 0ppm & $\begin{array}{c}\text { Germination Percentage (GP) } \\
\text { 200ppm }\end{array}$ & Speed of Germination Index (SGI) 200ppm \\
\hline \multirow{2}{*}{ V1 LOK-1 } & \multirow{2}{*}{100} & 44.49 & 37.76 \\
\cline { 2 - 4 } & \multirow{2}{*}{100} & $*(-55.5)$ & 31.99 \\
\cline { 2 - 4 } & \multirow{2}{*}{ V2 UP-2338 } & 40.19 & $*(-68.0)$ \\
\hline V3 PBW-154 & \multirow{2}{*}{100} & $*(-59.8)$ & 31.12 \\
\cline { 2 - 4 } & \multirow{2}{*}{100} & $*(-63.05)$ & $*(-68.87)$ \\
\hline V4 PBW-502 & \multirow{2}{*}{$*(-62.34)$} & 29.74 \\
\hline
\end{tabular}


Finally, comparing the tolerance behavior of four varieties of wheat to heavy metal concentration at the Critical level of 200ppm $\mathrm{Hg}$ as percent over control with percent decrease (-) over control on the basis of GP and SGI the four varieties showed the following trend (Table 13 and Graphs 12 \& 13).

\section{Discussion}

A variety of abiotic stresses including heavy metals causes molecular damage to plant cells either directly or indirectly through the formation of reactive oxygen species (ROS) $[57,58]$. Protonation of $\mathrm{O}_{2}{ }^{*}$ can produce the hydroperoxyl radical $\left(\mathrm{OH}^{*}, \mathrm{H}_{2} \mathrm{O}_{2}\right)$, which can convert fatty acids to toxic lipid peroxides, destroying biological membranes. The measurement of antioxidative enzymes could be useful for the level of antioxidant. The effects of heavy metals on plants resulted in growth inhibition, structural damage, a decline of physiological and biochemical activities, as well as of the function of plants. Our measurements showed that both growth and photosynthetic pigments are affected by the presence of heavy metals.

Rodriguez et al. [39] stated that mercury, a widely distributed environmental pollutant mainly supplied via anthropogenic sources to the soil, can induce toxicity in living organisms, including higher plants. Its toxicity, mobility, bioaccumulation, methylation process and transport in the atmosphere make Hg harmful and one of the best known toxic metals discharged from human activities. In the past couple of years the environmental protection agencies have expressed increasing concern over the release of mercury to the environment. Mercuric chloride $\left(\mathrm{HgCl}_{2}\right)$ is dominant among all mercury forms. Shriapanahi \& Anderson [59] found elevated levels of mercury in the upper layer of soil following long term application of municipal wastewater resulting in the accumulation of toxic levels of heavy metals in the vegetables grown in these areas.

The response of crop species to heavy metals reporting mechanisms responsible for their tolerance or sensitivity have been reported [59-64]. The toxic effect of mercury on germination, growth and yield has been studied on different plants [13,44,6568]. Parameters such as percentage of germination [69] and shoot and root lengths have been used as an indicator of heavy metal toxicity in plants $[70,71]$. Seeds treated with $\mathrm{Hg}$ showed lower germination percentage and that inhibition in seed germination at higher concentration of heavy metals was mainly caused by ion toxicity which is associated to changes in cellular permeability, inhibition of protein activity and/or direct toxicity to the embryo and seedling [72,73].

Reduction in germination of seeds in presence of $\mathrm{Hg}$ has been reported in rice [74] and in other plants [75-77]. The percentage of germination and \% DFC clearly indicate the inhibitory effects of $\mathrm{Hg}$ on germination. The results indicate that \%DFC increase with increasing concentration of metal solutions and higher values of $\%$ DFC suggest the greater susceptibility to Hg at higher concentration. In low level $\mathrm{Hg}$ concentration treatments, percentages germination were not much inhibited, showing that they were well within the tolerable range of seedlings. However, in high level treatments, germination percentages were detrimentally affected, implying that higher concentration of $\mathrm{Hg}$ was not conducive to seed germination. This may be attributed to depression of oxygen uptake and physiological disturbance in mobilization of reserve seed food materials [78].

Our results showed that seed germination of all the four wheat varieties was inhibited at all the concentrations of mercury as compared to controls. The degree of inhibition varied depending on the concentration of heavy metal. Seeing overall results it was recorded that the lowest germination percent (3.0\%) was found in $300 \mathrm{ppm}$ of $\mathrm{Hg}$ solution at 48 hours of germination in the variety V4 whereas highest $(94.0 \%)$ was recorded in Controls at 120 hours in the variety V3. It has been observed that the varieties V1 and V4 behaved better, even in 300ppm of $\mathrm{Hg}$ concentration at 120 hours of seedling growth. Thus, varieties show overall germination $\%$ as $-\mathrm{V} 1>\mathrm{V} 4>\mathrm{V} 3>\mathrm{V} 2$.

Seeds treated with $\mathrm{Hg}$ showed lower germination percentage. Several workers $[7,27,44,73]$ reported that inhibition in seed germination at higher concentration of heavy metals was mainly caused by ion toxicity. Ion toxicity is associated to changes in cellular permeability, inhibition of protein activity and/or direct toxicity to the embryo and seedling $[44,68]$. It has also been observed in Vigna ambacensis by Mohammad Nasser Al-Yemeni (2001). Reduction in germination of seeds in presence of $\mathrm{Hg}$ has been reported in rice [74] and in other plants [68,75-77]. Results also showed that V1 was more tolerant for heavy metal Hg in comparison to other wheat varieties.

\section{Conclusion}

Environmental Pollution has emerged as a major epidemic endangering Life on earth. Due to unwise, unscientific and excessive use of natural resources eco-balance is disturbed. Industrialization and urbanization have also deteriorated the position. As such 'pollution' can be considered the result of the growth of modern civilization. Hence 'effective Pollution control' is the need of the hour [7]. Episodes like the Minamata and Itai-Itai epidemics in Japan serve as a warning against the indiscriminate and careless use of toxic heavy metals. The concept that chemicals in the environment are transported through a cyclic process is not always re-assuring when one considers the time necessary for operations of the cycles to take place. These time lags may permit build up of specific chemicals to concentrations never before experienced.

Organisms, both plant and animal, have been shown to be capable of bio-accumulating toxic metals. Some of these enter the food chain and pose direct threat to man. One would like to know the complete natural history of occurrence, transport, transformation, accumulation and degradation for all toxic metals. Can environmental toxicology, combined with ecological considerations, provide information to prevent or minimize the hazards due to such events? A genuine appreciation of the problem of toxic metals in the environment is yet to be generated. There is no reason why with adequate understanding of the problem, one should not be able to continue to use toxic metals safely. It may be 
necessary to prescribe safe limits of exposure for the toxic metals and ensure that no exposure beyond these levels occur either occupationally or in the community.

The Water Pollution Act does not permit the discharge of toxic metals into water ways above certain limits. We are to ensure that these standards are adequately implemented. Similarly the atmospheric emission of toxic metals has also to be controlled below permissible limits that are likely to be recommended in the near future. For effective control of heavy metal pollution, it is necessary to continuously monitor the environment for their presence, to initiate a system for biological monitoring for heavy metal exposure and to take appropriate steps to minimize and control heavy metal pollution $[7,54]$.

Therefore, heavy metal pollutants are the main concern of new agricultural productions. Industrial products and using synthetic materials lead to drastically increase in concentration of different heavy metals in the environment. Heavy metals are largely used in electronic industries thus the wastewater of factories could pollute agricultural lands. Different heavy metal solutions were investigated for their effects on seed germination characteristics and phyto-remediation potential of a cereal crop (wheat). The wheat seeds germinated after treatments in solutions containing varying concentrations of heavy metal mercury showed that in all treatments the percentage of seed germination, root and shoot length decreased as concentrations of solution increased.

The present work compiling valuable material on Biological Control of Mercury Pollution supported by scientific experimental analysis of the issues and substantially based on the authoritative reference material derived from the writings of the eminent scientists in the field. The worker hopes that this work will prove an authoritative research work for students, scholars and academics in the field of heavy metal pollution besides policy planners, environmental scientists, laboratory technicians and environmental activists.

Thus, the objective of this work is to evaluate the toxicity of heavy metal mercury on wheat crop to provide information on the significance of seed germination rate, root/shoot ratio, dry and fresh weights of the seedlings, chlorophyll, carbohydrate contents, protein contents and oxidative enzymatic system of catalase, peroxidase and superoxide dismutase enzyme activities in response to heavy metal stress and to determine the effects of heavy metal $\mathrm{Hg}$ on growth and metabolism of crop plants [79-86].

\section{Recommendations}

In wheat (Triticum aestivum), Hg phytotoxicity contributed significantly towards reduction in percent seed germination. Planting crops in mercury-contaminated soil can produce significant health risks to consumers. Therefore, it is highly recommended that crops with short rooting systems should not be cultivated in mercury stress areas. Moreover a comprehensive public awareness through media and active participation of local youth is needed for avoiding such mercury induced toxicity problems in contaminated areas for growing agricultural crops.

\section{Acknowledgement}

The authors wish to thank Principal and Head Departments of Botany and Chemistry K.R. College, Mathura for kindly permitting and helping to work for the present investigation.

\section{References}

1. Yadav, Poonam (2011) Evaluation of phyto-toxicity and relative tolerance of plants to air pollutants.

2. Pandey, Tripti (2012) Impact of some air pollutants on vegetation in Varanasi City.

3. Pandey, Tripti, Nidhi Parashar, Ravi Sharma (2007) Biochemical changes in Azadirachta indica and Carica papaya with respect to air pollution. Plant Archives 7(2): 689-691.

4. Sharma, Ajeet Kumar (2004) Water quality problem in Mathura and its effect on the physiology of major crops in the region.

5. Singh, Shalini (2009) Relative tolerance of crop plants to heavy metal pollution.

6. Gautam, Shelja (2010) Study of phytotoxicity of heavy metal pollution and its control.

7. Parashar, Nidhi (2011) Planning and Investigation for City and Industrial Effluent Utilization in Abating Pollution of River Yamuna and Improving Agricultural Production.

8. Sharma, Ravi (1976) Environmental Problems in Mathura; Workshop on Environmental Education for Asian Youth, INSA, New Delhi \& Nainital (UP) 22-30 June, Proc Indian Environmental Society 56: 1

9. Sharma, Ravi (1982) Physiology of plant tolerance to salinity at early seedling stage.

10. Sharma, Ravi (1987) Towards an understanding of the physiology of salt tolerance in wheat (Triticum aestivum L.) at early seedling stage.

11. Nauhbar, Suman (2005) Relative tolerance of crop plants to salt stress at the early seedling stage.

12. Yadav (2006) Physiology of salt tolerance for effective biological control of salinity.

13. Rani, Saroj (2007) Investigations on salt tolerance parameters specially growth and biochemical traits for selection of salt tolerant lines in legumes at the early seedling stage.

14. Pathak, Abha (2017) Differential growth and metabolic behavior of cereal and legume crops under the influence of heavy metal cadmium.

15. Singh, Chandra Bhan (2015) Use of Chemical Pesticides for Commercial Production of Vegetables and Their impact on the Environs in the Village Manoharpur, Mathura-A Case Study.

16. Snehlata (2016) Utilization of Sewage Effluents for Irrigating Crop Plants for Their Sustainable Improvement with special Reference to Mathura.

17. Nriagu JO, Pacyna JM (1988) Quantitative assessment of worldwide contamination of air water and soils by trace metals. Nature 333(6169): 134-139.

18. Schalscha E, Ahumada I (1998) Heavy metals in rivers and soils of central chile. Water Sci Technol 37(8): 251-255.

19. McGrath MJ, Luyssaert S, Meyfroidt P, Kaplan JO, Bürgi M, et al. (2010) Reconstructing European forest management from 1600 to 2010. Biogeosciences 12: 4291-4316,

20. Foy CD, Chaney RL, Fleming AL, White MC (1978) The physiology of metal toxicity in plants. Annu Rev Plant Physiol 29: 511- 566.

21. Mehera A, Farago ME (1994) Plants and Chemical Elements: Biochemistry Uptake Tolerance and Toxicity. 
22. Vangronsveld J, Clijsters H (1994) Toxic effect of metals. In: Farago ME (Ed.), Plants, Plants and Chemical Elements. pp: 149-175.

23. Prasad MNV (1997) Trace elements. In: Prasad MNV (Ed.), Plant Ecophysiology. John Wiley \& Sons, USA, pp: 207-249.

24. Srivastava AK, Purnima (1999) Some facets of heavy metal tolerance in higher plants. J Indian bot Soc 78: 271-286.

25. Garbisu C, Alkorta I (2001) Phytoextraction: A cost effective plant-based technology for the removal of metals from the environment. Biores Technol 77(3): 229-236.

26. Gisbert C, Ros R, de Haro A, Walker DJ, Pilar Bernal M, et al. (2003) A plant genetically modified that accumulates $\mathrm{Pb}$ is especially promising for phytoremediation; Biochem Biophys Res Commun 303(2): 440-445.

27. Pathak A, Parashar SN, Snehlata, Saraswat K, Sharma R (2017) Differential Growth and Metabolic Behaviour of Cereal and Legume Crops under the Influence of Heavy Metal Cadmium: I-Germination Percentage (GP) and Speed of Germination Index (SGI). SF J Plant Physiol 1(1): 1-22.

28. Saraswat, Kapila (2017) Studies on the phyto-toxicity of heavy meta mercury on germination, early seedling growth and biochemical changes in wheat (Triticum aestivum L.).

29. Sharma, Ravi (2017) Genotypic response to salt stress II-Pattern of Differential Relative Behavior of Salt-tolerant, Moderately Salt-Tolerant and Salt-Sensitive Wheat cultivars under Salt Stressed Conditions. Inter Jour Gr Sed \& Water.

30. Smith WE, Smith AM (1975) Minamata Health Reinhart and Winston New York.

31. Ramanthan NL (1980) Toxic metals and human health; Key Note Address for Indian Environmental Society Seminar on World Environment Day. pp: $1-16$.

32. Rytuba, James J (2002) Mercury from mineral deposits and potential environmental impact. Environ Geol 43(3): 326-338.

33. Cocoros G, Cahn PH, Siler W (1973) Mercury concentrations in fish, plankton and water from three Western Atlantic estuaries. Jour Fish Biol 5(6): 641-647.

34. McGrath SP, Zhao FJ, Lombi F (2001) Plant and rhizosphere process involved in phytoremediation of metal-contaminated soils. Plant Soil 232(1-2): 207-214

35. Gade LH (2000) Highly polar metal-metal bonds in earlylate heterodimetallic complexes; Angewandte ChemieInternational 3915(edn), pp: 2658-2678.

36. McKeehan, Brownfields P (2000) The Financial, Legislative and Socia Aspects of the Redevelopment of Contaminated Commercial and Industrial Properties 2: 178-191.

37. Ragnarsdottir KV, Hawkins D (2005) Trace metals in soils and their relationship with scrapie occurrence. Geochimica et Cosmochimica Acta 69(10): A196-A196.

38. Liu Y (2006) Shrinking Arable Lands Jeopardizing China's Food Security.

39. Rodriguez E, Santos C, Azevedo R, Moutinho Pereira J, Correia C, et al. (2003) Chromium (VI) induces toxicity at different photosynthetic levels in pea. Plant Physiology and Biochemistry 53: 94-100.

40. Patra M, Sharma A (2000) Mercury toxicity in plants. Bot Rev 66: 379422.

41. Goyer RA, Clarkson TW (2001) Toxic effects of metals. In: Klaasen CD (Ed.), Casarett and Doullis Toxicology: The Basic Science of Poisons. McGraw-Hil, New York, USA.

42. Cao LA, Zhang Q, Kong XXu, Josine TL, Chen X (2007) Over expression of GLT1 in fps1DeltagpdDelta mutant for optimum ethanol formation by Saccharomyces cerevisiae. Biomol Eng 24(6): 638-642.

43. Liu ZL, Goh SH, Ho SH (2007) Screening of Chinese medicinal herbs for bioactivity against Sitophilus zeamais Mostchulsky and Tribolium castaneum (Herbst). J Stored Prod Res 43: 290-296.

44. Singh DK, Nidhi Parashar, Ravi Sharma (2008) Physico-chemical analysis of effluents from a carpet industry. Plant Archives 8(2): 577-583.

45. Yannarelli GG, Fernándezm Alvarez AJ, Santa Cruz DM, Tomaro ML (2007) Glutathione reductase activity and isoforms in leaves and roots of wheat plants subjected to cadmium stress. Phytochemistry 68: 505512 .

46. Lindberg AL, Kumar R, Goessler W, Thirumaran R, Gurzau E, et al. (2007) Metabolism of low-dose inorganic arsenic in a central European population: influence of sex and genetic polymorphisms. Environ Health Perspect 115: 1081-1086

47. Sun J, Yu EY, Yang Y, Confer LA, Sun SH, et al. (2009) Stn1-Ten1 is an Rpa2-Rpa3-like complex at telomeres. Genes Dev 23(24): 2900-2914.

48. Groppa MD, Benavides MP, Tomaro ML (2003) Polyamine metabolism in sunflower and wheat leaf discs under cadmium or copper stress. Plant Sci 164: 293-299.

49. Schmidt U (2003) Enhancing phytoremediation: The effect of chemical soil manipulation on mobility, plant accumulation, and leaching of heavy metals. J Environ Qual 32: 1939-1954.

50. Schwartz C, Echevarria G, Morel JL (2003) Phytoextraction of cadmium with Thlaspi caerulescens. Plant Soil 249(1): 27-35.

51. Garrard A (1945) The effect of b-indolyl acetic acid on the germination and root growth of certain members of Cruciferae; New Phytol 53(2): 165-176.

52. Sarin MN, Rao IM (1956) Effect of sodium sulphate on early seedling growth of gram and wheat; Agra Univ J Res Sci 5(1): 143-154.

53. Sheoran IS, Garg OP (1978) Effect of salinity on the activities of RNase, DNase and protease during germination and early seedling growth of mung bean. Physiol Plant 44(3): 171-174.

54. Sharma S, Yang J, Watzinger P, Kötter P, Entian KD, et al. (2013) Yeast Nop2 and Rcm1 methylate C2870 and C2278 of the 25S rRNA, respectively. Nucleic Acids Res 41(19): 9062-9076.

55. Sharma, Ravi 2015 Genotypic response to salt stress: I-Relative tolerance of certain wheat cultivars to salinity. Adv Crop Sci Tech 3(4).

56. Carley HE, Watson RD (1968) Effect of various aqueous extract upon seed germination. Bot Gaz 129: 57-62.

57. Greger M, Kautsky L, Sandberg T (1995) A tentative model of Cd uptake in Potamogeton pectinatus in relation to salinity. Environ-mental and Experimental Botany 35(2)L: 215-225.

58. Lin CC, Kao CH (2000) Effect of $\mathrm{NaCl}$ stress on $\mathrm{H}_{2} \mathrm{O}_{2}$ metabolism in rice leaves. Plant Growth Regul 30: 151-155.

59. Shriapanahi, Anderson, Shriapanahi M, Anderson AC (1986) Accumulation of $\mathrm{Cd}, \mathrm{Hg}, \mathrm{Pb}$ by vegetables following long-term land application of waste water. Sci Total Environ 52: 41-48.

60. Ernest W (1976) Physiological and biochemical aspects of metal tolerance; In: Mansfield TA (Ed.), Effect of Air Pollutants on Plants, Cambridge University Press, London, UK, England, pp: 115-133.

61. Williams, Gary M, Robert Kroes, Ian C, Munro (2000) Safety Evaluation and Risk Assessment of the Herbicide Roundup and Its Active Ingredient, Glyphosate, for Humans Regulatory Toxicology and Pharmacology 31: 117-165.

62. Watanabe, Toshihiro, Mitsuru Osaki (2002) Mechanisms of adaptation to high aluminum condition in native plant species growing in acid soils: a review 33: 1247-1260.

63. Mahmood S, Hassan S, Ahmed F, Ashraf M, Alam M, et al. (2005) Influence of feed withdrawal for different durations on performance of broilers in summer. Int J Agri Biol 7: 975-978. 
64. Vitorello, Capaldi VA, Stefanuto (2005) Recent advances in aluminum toxicity and resistance in higher plants. Braz J of Plant Physiol 17: 129143.

65. Vizarova G, Zatkalikova T, Zelenakoua E (1985) Effects of Hg on some physiological processes in barley. Biologia 50: 573-576.

66. Varshney KA (1991) Phytotoxic effects of mercuric acetate on the development of chlorophyll in excised cotyledons of two cucurbits. Geobios 18: 119-124.

67. Mukherjee AK, De B (1996) Hg induced metabolic changes in seedlings of cultivated cells of tomato. Geobios 23: 83-88.

68. Gautam, Aruna (2009) The problem of saline wastelands and their management-A Biological Approach with special reference to Mathura.

69. Mhatre GN, Chaphekar SV (1984) Response of young plants to mercury. Water, Soil and Air Pollution 21: 1-8.

70. Uveges JL, Corbett AL, Mal TK (2002) Effects of lead contamination on the growth of Lythrum salicaria (purple loosestrife); Environ. Pollut 120: 319-323.

71. Prasad KVSK, Pardha Saradhi P, Sharmila P (1999) Concerted action of antioxidant enzymes and curtailed growth under zinc toxicity in Brassica juncea; Environ Expt Bot 42: 1-10.

72. Dubey RC, Dwivedi RS (1987) Effect of heavy metals on seed germination and seedling growth of soybean. Nat Acad Sci Lett 10(4): 121-123.

73. Iqbal MZ, Mohamed MT, Ahmed F (1991) Influence of cadmium toxicity on germination and growth of some common trees; Pak J Sci Indust Res 34: $140-142$.

74. Mukherjee S, Maitra P (1976) Toxic effects of lead on growth and metabolism of germinating rice seeds; Indian J Exp Biol 14: 519-521.

75. Kacabove P, Nar L (1986) Effect of lead on growth characteristics and chlorophyll content in barely seedlings; Photosynthetic. 20: 411-417.
76. Mesenon MN, Jaber K (1991) The toxic effect of lead on seed germination, growth, chlorophyll and protein content of wheat. Acta Hung 42: 331344.

77. Hsu FH, Chang HC (1992) Inhibitory effects of the heavy metals on seed germination and seedling growth of Miscanthus species. Bot Bull Acad 33: 335-342.

78. De Andrade, Sara AL, Adraina PD, da Silveira (2008) Mycorrhiza influence on maize development under Cd stress and P supply Brazl J Plant Physiol 20(1): 39-50.

79. Baker AJM, Brooks RR (1989) Terrestrial higher plants which hyperaccumulate metallic elements-a review of their distribution, ecology and phyto chemistry. Bio recovery 1: 81-126.

80. EPA (2013) Advancing Sustainable Materials Management: Fact Sheet Assessing Trends in material generation Recycling and Disposal in the US 2015.

81. Liu, Zhang, Liu Z, Carbrey JM, Agre P, et al. (2007) Arsenic trioxide uptake by human and rat aquaglyceroporins. Biochem Biophys Res Commun 316: 1178-1185.

82. Liu Z, Shen J, Carbrey JM, Mukhopadhyay R, Agre P, et al. (2002) Arsenite transport by mammalian aquaglyceroporins AQP7 and AQP9. Proc Natl Acad Sci USA 99: 6053-6058.

83. Mohammed Nasser Al Yemeni (2001) Effect of cadmium, mercury and lead on seed germination and early seedling growth of Vigna ambacensis L. Ind J Plant Physiol 6: 147-151.

84. Pandey, Tripti, Nidhi Parashar, Ravi Sharma (2007) Seasonal variation in the ambient air quality of a tropical city Varanasi, India-a case study. Plant Archives 7(2): 821-823.

85. Prasad MNV (1995) Cadmium toxicity and tolerance in vascular plants-A review. Enu Exp Bot 35: 525-545.

86. SPSS Inc (2003) Statistics, SPSS Inc Chicago. USA.
Creative Commons Attribution 4.0 International License

For possible submissions Click Here

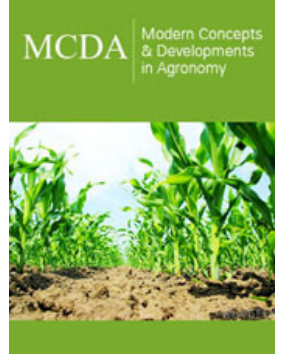

\section{Modern Concepts \& Developments in Agronomy}

\section{Benefits of Publishing with us}

- High-level peer review and editorial services

- Freely accessible online immediately upon publication

- Authors retain the copyright to their work

- Licensing it under a Creative Commons license

- Visibility through different online platforms 